\title{
The role of the turbulent scales in the settling velocity of heavy particles in homogeneous isotropic turbulence
}

\author{
By C. Y. YANG AND U. LEI $\dagger$ \\ Institute of Applied Mechanics, National Taiwan University, Taipei 106, Taiwan, \\ Republic of China
}

(Received 18 April 1997 and in revised form 21 April 1998)

The average settling velocity of heavy particles under a body force field is studied numerically in stationary homogeneous isotropic turbulent flows generated by the direct numerical simulation and the large eddy simulation of the continuity and Navier-Stokes equations. The flow fields corresponding to different selected ranges of turbulent scales are obtained by filtering the results of the direct numerical simulation, and employed for calculating the particle motion. Wang \& Maxey (1993) showed that as a consequence of the particle accumulation in the low vorticity region and the preferential sweeping phenomenon, the average settling rate in turbulence is greater than that in still fluid. In the present study, the phenomenon of particle accumulation in the low vorticity region is found to be controlled mainly by the small scales with wavenumber $k_{\omega}$ corresponding to the maximum of the dissipation (vorticity) spectrum. However, the increase of the average settling rate, $\left\langle\Delta v_{S}\right\rangle$, also depends strongly on the large energetic eddies. The small eddies with wavenumber greater than $2.5 k_{\omega}$ have essentially no effect on the particle accumulation and the average settling velocity. The large eddy simulation is thus adequate for the present study provided the smallest resolved scale is greater than $1 /\left(2.5 k_{\omega}\right)$. Detailed calculations show that $\left\langle\Delta v_{S}\right\rangle$ is maximized and is of order $u^{\prime} / 10$ when $\tau_{p} / \tau_{k} \approx 1$ and $v_{d} / u^{\prime} \approx 0.5$ for $R_{\lambda}=22.6-153$, where $\tau_{p}$ is the particle's relaxation time, $\tau_{k}$ is the Kolmogorov timescale, $v_{d}$ is the settling rate in still fluid, $u^{\prime}$ is the root mean square of the velocity fluctuation, and $R_{\lambda}$ is the Reynolds number based on the Taylor microscale.

\section{Introduction}

Knowledge of the ensemble-average settling rate of small heavy particles in turbulent flow is important for many applications in environmental science and engineering. Examples include the settling of aerosol particles in the atmosphere and water droplets in clouds. Consider the settling of heavy spherical particles in homogeneous isotropic turbulence with zero mean flow. It was generally assumed before the 1980s that the ensemble average of the settling velocity of particles in turbulence, $\left\langle\boldsymbol{v}_{S}\right\rangle$, equals the settling velocity in still fluid, $\boldsymbol{v}_{d}$. For example, Reeks (1977) has argued that $\left\langle v_{S}\right\rangle=v_{d}$ if the flow field is treated as a stochastic random field, where $\left\langle v_{S}\right\rangle$ and $v_{d}$ are the magnitudes of $\left\langle\boldsymbol{v}_{S}\right\rangle$ and $\boldsymbol{v}_{d}$, respectively. Here the directions of $\left\langle\boldsymbol{v}_{S}\right\rangle$ and $\boldsymbol{v}_{d}$ are the same as the direction of the body force field. Maxey (1987) showed that $\left\langle v_{S}\right\rangle$ is indeed equal to $v_{d}$ for zero-inertia particles through a rigorous analysis. For large-inertia

$\dagger$ Author to whom the correspondence should be addressed. 
particles, the particle aerodynamical response time is much greater than any integral timescale of the flow; the turbulent fluid velocity seen by a particle appears as an uncorrelated random noise and should have negligible effect on the settling velocity. For particles with finite inertia, Maxey showed that $\left\langle v_{S}\right\rangle$ is greater than $v_{d}$ in a Gaussian random flow field. The effect of the dynamics of turbulence was included by Yeh \& Lei (1991), and they also found that $\left\langle v_{S}\right\rangle$ is greater than $v_{d}$ according to a simulation of the particle motion in a decaying homogeneous isotropic turbulent flow field generated by large eddy simulation (LES). However, the increase of the magnitude of the average settling velocity, $\left\langle\Delta v_{S}\right\rangle$, obtained by Yeh \& Lei is smaller than that obtained by Maxey (1987). Here $\left\langle\Delta v_{S}\right\rangle=\left\langle v_{S}\right\rangle-v_{d}$. Recently, Wang \& Maxey (1993) studied the settling of particles in a stationary homogeneous isotropic turbulence generated by the direct numerical simulation of the continuity and Navier-Stokes equations (DNS), and found substantially greater values of $\left\langle\Delta v_{S}\right\rangle$ in comparison with those obtained by Yeh \& Lei. A significant increase in $\left\langle\Delta v_{S}\right\rangle$ can occur for particles with inertial response time $\left(\tau_{p}\right)$ and $v_{d}$ comparable to the Kolmogorov scales of the turbulence. Wang \& Maxey pointed out that the flow field generated by LES like that in Yeh \& Lei is not adequate for evaluating the present problem. The reason is that the particle accumulation in the low vorticity region (one of the key mechanisms for the increase of the settling velocity) is related to the small scales of turbulence, which are not resolved in LES. According to their results, Wang \& Maxey also suggested that the Kolmogorov scaling is important for the phenomenon associated with the increase of settling velocity, which is interesting and essentially correct, but is in contrast to the traditional approach that the large-scale, energy-containing fluid motions dominates the transport of particles.

According to the direct numerical simulations of homogeneous turbulence (for examples, see Vincent \& Meneguzzi 1991; Jiménez et al. 1993), the vorticity structure of the flow is found to be organized in coherent, cylindrical or ribbon-like, vortices, which are also known as 'worms' according to Jiménez et al. A statistical study by Jiménez et al. suggests that the vorticies are simply especially intense features of the background vorticity. The radii and the lengths of the vorticies are of the order of the Kolomogorov and integral length scales $\left(\eta\right.$ and $\left.L_{f}\right)$, respectively. The spacing between the vorticies is of order $\lambda$, the Taylor microscale (Vincent \& Meneguzzi). Jiménez et al. also found that the circulation of the intense vorticies, $\gamma$, increases as $v R_{\lambda}^{0.5}$, where $R_{\lambda}=u^{\prime} \lambda / v$ is the Reynolds number based on the Taylor microscale, and $v$ is the kinematic viscosity. Here $u^{\prime}$ is the root mean square of the velocity fluctuation, which is also the velocity scale associated with the large energetic eddies. Let $U_{S}$ be the velocity scale outside the intense vorticies. With $\gamma \sim U_{S} \eta$ and $\eta / \lambda \sim R_{\lambda}^{-0.5}$, it follows that $U_{S} \sim u^{\prime}$, which indicates that the velocity scale in the low vorticity regions between the intense vortices ('worms') is of order $u$ '.

As the diameter of the particles, $d_{p}$, is in general of one order less than the Kolmogorov length scale, the motion of a particle is exposed to the action of the whole range of the length scales of turbulence. When the particles settle under gravity (or another body force field) through the flow field, the particle inertia produces a bias in each trajectory towards regions of low vorticity due to the local centrifugal effect of the tubular vortical structures ('worms'), and thus the particles tend to accumulate in the low vorticity (or high strain rate) region. This phenomenon of particle accumulation was simulated by Squires \& Eaton (1991) and Wang \& Maxey (1993). The degree of particle accumulation depends on the particle inertia, and is maximized at a certain finite value in a given flow field under the action of a specified body force. An instantaneous distribution of particles in a velocity field is 
shown in figure 12 of Wang \& Maxey (1993). It can be observed clearly from the figure that there are essentially no particles in the cores of the vortices (high vorticity region), but many particles are collected in elongated sheets on the peripheries of local vortical structures (low vorticity region). Furthermore, in the low vorticity region, more particles are accumulated at the downward side (with the angle between the local fluid velocity and the body force, $\theta$, less than $90^{\circ}$ ) in comparsion with the upward side $\left(90^{\circ}<\theta<180^{\circ}\right)$, which is called preferential sweeping by Wang \& Maxey. As the local drag on the particle is less at the downward side and more particles are there, the ensemble average of the settling velocity of particles is thus increased.

Although the fundamental framework, including the key results and the underlying physics, of the increase of the average settling velocity of particles in homogeneous stationary isotropic turbulence has been completed successfully by Maxey (1987) and Wang \& Maxey (1993), the roles of different turbulent scales are still unclear and worth further investigation. The reasons are as follows:

(1) The accumulation of particles in the low vorticity region is related strongly to the structure of the tubular vortices (of diameter $\eta$ approximately), or the details of the instantaneous vorticity field. This is probably the main reason that Wang \& Maxey employed DNS to generate their flow field. However, the length scale corresponding to the maximum of the vorticity (dissipation) spectrum, $l_{\omega}$, is in general of one order greater than the Kolmogorov length scale, $\eta$ (see Tennekes \& Lumley 1972 for example). Also the vorticity associated with $\eta$ is of one order less than that associated with $l_{\omega}$, and the energy associated with the Kolmogorov eddies are negligible. It is possible that the Kolmogorov eddies might have a significant effect on the accumulation of particles, and hence provide a contribution to the increase of the settling velocity. Here $l_{\omega}=1 / k_{\omega}$, with $k_{\omega}$ the wavenumber corresponding to the maximum of the dissipation spectrum. From the numerical point of view, should one always need to resolve accurately the Kolmogorov eddies for the correct estimation of the increase of the settling velocity? If the Kolmogorov eddies are essentially not important, one may still employ the flow field generated by large eddy simulation (LES) with suitable cutoff length scales (smaller than those in Yeh \& Lei 1991) to study the average settling velocity.

(2) Although Wang \& Maxey found a substantial increase of the average settling velocity when $v_{d} \approx v_{k}$ (the Kolmogorov velocity scale) and $\tau_{p} \approx \tau_{k}$ (the Kolmogorov time scale), and demonstrated the importance of the Kolmogorov scaling for the present problem, the role of the large eddies deserves further examination. As the particles are accumulated in the region of low vorticity where the velocity scale of the flow is $u^{\prime}$, it is expected that the large energetic eddies should play a significant role on the drag and thus the increase of the average settling velocity. Note that the Kolmogorov time scale, $\tau_{k}$, proposed by Wang \& Maxey is indeed a suitable time scale for the present problem. The reason is that $\tau_{k}^{-1}$ is also the scale for the vorticity corresponding to the 'eddies' associated with $l_{\omega}$, which are responsible for the particle accumulation.

The major work of the present paper is to carry out a detailed and careful study on the effects of different length scales on the average settling velocity of particles in stationary homogeneous isotropic turbulence generated by DNS and LES. The paper is organized as follows. The formulation and numerical method are described in $\S 2$. The results for stationary turbulence are presented and discussed in $\S 3$. The validity of the present calculation is first checked against the result by Wang \& Maxey. Effects of small and large scales are then studied in detail. The validity of using the flow field generated by LES with suitable cutoff wavenumber for studying the settling velocity 
is checked against the results using DNS. LES is then employed to extend the range of $R_{\lambda}$ (which possesses a short inertial subrange) of the flow field for the present study. Detailed effects of parameters on $\left\langle\Delta v_{S}\right\rangle$ are presented, and the scales for the present problem are discussed. Finally, the paper is concluded in $\S 4$.

\section{Formulation and numerical method}

\subsection{Formulation}

Consider an incompressible Newtonian fluid with density $\rho$ and constant kinematic viscosity $v$. The governing equations for the homogeneous isotropic turbulent flow field in an inertial frame, written in index form, are

$$
\begin{gathered}
\frac{\partial u_{i}}{\partial x_{i}}=0 \\
\frac{\partial u_{i}}{\partial t}+\frac{\partial\left(u_{i} u_{j}\right)}{\partial x_{j}}=-\frac{1}{\rho} \frac{\partial p}{\partial x_{i}}+v \frac{\partial^{2} u_{i}}{\partial x_{j} \partial x_{j}}+f_{i}
\end{gathered}
$$

where $u_{i}(i=1,2,3)$ are the velocity components, $t$ is the time, $x_{i}(i=1,2,3)$ are the spatial coordinates, $p$ is the pressure, and $f_{i}$ is the forcing term for maintaining the turbulence stationary. The motion of a single spherical solid particle with diameter $d_{p}$ and mass $m_{p}$ in the turbulent gas flow field is assumed to follow

$$
m_{p} \frac{\mathrm{d} \boldsymbol{v}}{\mathrm{d} t}=3 \pi d_{p} \rho v C(\boldsymbol{u}-\boldsymbol{v})+m_{p} \boldsymbol{g},
$$

and the particle's trajectory is evaluated by

$$
\frac{\mathrm{d} \boldsymbol{Y}}{\mathrm{d} t}=\boldsymbol{v},
$$

where $\boldsymbol{Y}(t)$ and $\boldsymbol{v}(t)$ are the instantaneous displacement and velocity of the particle at time $t, \boldsymbol{u}=\boldsymbol{u}(\boldsymbol{Y}(t), t)$ is the instantaneous fluid velocity where the particle is located, $\boldsymbol{g}$ is the body force per unit mass of the particle, and $C$ is a factor accounting for the inertial effect of the drag term. In the present study, we set $C=1+0.1315 R_{p}^{0.82-0.05 \log _{10} R_{p}}$, which is an experimental correlation for the standard drag curve of a single sphere in a uniformly moving stream, and is valid for $0.01 \leqslant R_{p} \leqslant 20$ (see Clift, Grace \& Weber 1978, p. 112). Here $R_{p}=|\boldsymbol{u}-\boldsymbol{v}| d_{p} / v$ is the particle's Reynolds number. The settling velocity in still fluid $\boldsymbol{v}_{d}=\boldsymbol{g} \tau_{p} / C_{0}$, where $\tau_{p}=m_{p} /\left(3 \pi d_{p} \rho v\right)$ is the particle's relaxation time (inertial response time) based on the Stokes drag law, and $C_{0}$ is the value of $C$ evaluated with $\boldsymbol{u}=0$. The added mass effect, the Basset force, and the buoyancy force are all neglected in $(2 a)$ since the density of the particle, $\rho_{p}$, is much greater than the fluid density, $\rho$. Here we set $\rho_{p} / \rho=1000$ in the calculations. In the drag term of $(2 a), \boldsymbol{u}$ is obtained by solving the transient three-dimensional governing equations $((1 a)$ and $(1 b))$ using either DNS or LES. Motions of thousands of particles are simulated numerically by solving $(2 a)$ and $(2 b)$, and employed for studying the ensemble average of the particle properties.

\subsection{Numerical method using DNS}

The method and procedures employed here for solving the flow field are basically the same as those in Lee \& Reynolds (1985), which are based on Rogallo (1977, 1981). Such procedures have also been employed and extended successfully by Yeh $\&$ Lei (1991) for simulating the particle motion in a decaying homogeneous isotropic 
turbulence generated by LES. For homogeneous isotropic turbulence, the calculation domain for $(1 a)$ and $(1 b)$ is a cube with edge length $L$ subject to the periodic boundary conditions. Here $L$ should be sufficiently larger than the separation at which two-point spatial correlations vanish and the length scale associated with the particle motion, $v_{d} \tau_{p}$ (see Yeh \& Lei 1991). In accordance with the periodic boundary conditions, the velocity and pressure fields are expanded by three-dimensional discrete Fourier series with $N$ wavenumbers in any direction. The linear spatial terms in $(1 a)$ and $(1 b)$ are evaluated easily by direct differentiation. The pseudospectral method was used for evaluating the nonlinear term $\partial\left(u_{i} u_{j}\right) / \partial x_{j}$. The aliasing problem is handled by using the hybrid dealiasing algorithm by Lee \& Reynolds (1985), which is modified from the hybrid method proposed by Rogallo $(1977,1981)$. The doubly and triply aliased terms are eliminated by using a spherical truncation mask set at $k^{2}=\frac{2}{9} N^{2}$ following Patterson \& Orszag (1971). The singly aliased term is handled by the random phase shift method of Rogallo (1977, 1981). The forcing term in the wavenumber space, $\hat{f}_{i}(\boldsymbol{k}, t)$, is evaluated similar to Eswaran \& Pope (1988). To guarantee the continuity condition,

$$
\hat{f}_{i}(\boldsymbol{k}, t)= \begin{cases}\hat{a}_{i}-k_{i}\left(\hat{a}_{j} k_{j} / k^{2}\right), & \text { if } 0<k<k_{f c} \\ 0, & \text { otherwise, }\end{cases}
$$

where $\boldsymbol{k}\left(k_{1}, k_{2}, k_{3}\right)$ is the wavenumber, $k_{f c}$ is the forcing radius in the wavenumber space, and $\hat{a}_{i}$ is assumed to follow

$$
\frac{\mathrm{d} \hat{a}_{i}}{\mathrm{~d} t}+\frac{\hat{a}_{i}}{T_{f c}}=S_{f c} R_{i}(t) A_{i} \quad \text { for } \quad 0<k<k_{f c},
$$

with the factor

$$
A_{i}=\exp \left[-10\left(\frac{u_{i}^{2}}{q^{2}}-\frac{1}{3}\right)\right], \quad q^{2}=u_{1}^{2}+u_{2}^{2}+u_{3}^{2}, \quad i=1,2,3 .
$$

Here $A_{i}$ is employed for reducing the anisotropic effect due to the articifical forcing, $R_{i}(t)$ is a random function distributed uniformly between -0.5 and $0.5, S_{f c}$ is the forcing amplitude, and $T_{f c}$ is the forcing time scale. In the present study, we choose $k_{f c}=\sqrt{8}$, which is the same as that in Wang \& Maxey (1993). The values of $S_{f c}$ and $T_{f c}$ employed in the present study are listed in table 1, and will be discussed in $\S 3$. Finally, $(1 a)$ and $(1 b)$ become a set of ordinary differential equations in the wavenumber space, which are solved for $\hat{\boldsymbol{u}}$, the Fourier transform of the velocity. The initial three-dimensional, incompressible and isotropic field is constructed using the method in Yeh \& Lei (1991). An initial energy spectrum

$$
E(k)=\frac{8}{\pi} u_{0}^{\prime 2} L_{f 0}^{2} \frac{k^{4}}{\left(1+k^{2} L_{f 0}^{2}\right)^{3}}
$$

is employed for starting the calculation provided $u_{0}^{\prime}$ and $L_{f 0}$ are specified. An inverse transform of $\hat{\boldsymbol{u}}$ gives the flow field in the physical space, $\boldsymbol{u}$, which is required for the drag term in $(2 a)$ for the particle simulation. In order to study the effect of different scales of turbulence on the particle's settling velocity, certain modes in the wavenumber domain can be selected for calculating the particle motion using a numerical 'Fourier space sharp-cutoff filter' during the inverse Fourier transform. An example is illustrated as follows. Let $k_{\max }, k_{\min }, k_{C H}$ and $k_{C L}$ be the magnitudes of the highest simulated wavenumber, the lowest simulated wavenumber, the high end cutoff wavenumber of the filter, and the low end cutoff wavenumber of the filter, respectively. If $k_{C H}<k_{\max }$ and $k_{C L}=k_{\min }$, the modes for the range $k_{C H}<k<k_{\max }$ of the simulated 


\begin{tabular}{|c|c|c|c|c|c|c|}
\hline & Case A & Case B & Case C & Case D & Case E & Case F \\
\hline$L^{3}$ & $(2 \pi)^{3}$ & $(2 \pi)^{3}$ & $(2 \pi)^{3}$ & $(2 \pi)^{3}$ & $(2 \pi)^{3}$ & $(2 \pi)^{3}$ \\
\hline$N^{3}$ & $32^{3}$ & $64^{3}$ & $96^{3}$ & $32^{3}$ & $96^{3}$ & $128^{3}$ \\
\hline Method & DNS & DNS & DNS & LES & LES & LES \\
\hline$S_{f c}$ & 180 & 180 & 180 & 180 & 180 & 180 \\
\hline$T_{f c}$ & 0.2 & 0.18 & 0.195 & 0.195 & 0.2 & 0.2 \\
\hline$u_{f 0}^{\prime}$ & 3.0 & 3.0 & 3.0 & 3.0 & 3.0 & 3.0 \\
\hline$L_{f 0}$ & 1.5 & 1.5 & 1.5 & 1.5 & 1.5 & 1.5 \\
\hline$v$ & 0.15 & 0.08 & 0.03 & 0.03 & 0.0075 & 0.005 \\
\hline$u^{\prime}$ & 3.35 & 3.14 & 3.16 & 3.10 & 3.10 & 2.95 \\
\hline$L_{f}$ & 1.62 & 1.55 & 1.60 & 1.65 & 1.23 & 1.08 \\
\hline$\epsilon$ & 25.2 & 14.4 & 11.8 & 9.68 & 10.5 & 9.7 \\
\hline$S$ & -0.47 & -0.47 & -0.51 & -0.42 & -0.44 & -0.44 \\
\hline$T_{e 1}$ & 0.46 & 0.49 & 0.51 & 0.53 & 0.40 & 0.37 \\
\hline$T_{e 2}$ & 0.45 & 0.68 & 0.85 & 0.99 & 0.91 & 0.90 \\
\hline$\eta$ & 0.108 & 0.078 & 0.039 & 0.041 & 0.014 & 0.011 \\
\hline$\tau_{k}$ & 0.077 & 0.075 & 0.05 & 0.055 & 0.027 & 0.023 \\
\hline$v_{k}$ & 1.39 & 1.04 & 0.77 & 0.74 & 0.53 & 0.47 \\
\hline$\lambda^{n}$ & 1.01 & 0.91 & 0.62 & 0.68 & 0.32 & 0.26 \\
\hline$R_{\lambda}$ & 22.6 & 35.7 & 65.3 & 69.8 & 133 & 153 \\
\hline$k_{\max } \eta$ & 1.62 & 1.7 & 1.71 & 0.61 & 0.62 & 0.63 \\
\hline$u^{\prime} / v_{k}$ & 2.41 & 3.02 & 4.10 & 4.21 & 5.85 & 6.28 \\
\hline$L_{f} / \eta$ & 15 & 20 & 41 & 40 & 87 & 102 \\
\hline$L_{f} / \lambda$ & 1.60 & 1.70 & 2.58 & 2.44 & 3.84 & 4.15 \\
\hline$T_{e 1} / \tau_{k}$ & 5.97 & 6.63 & 10.1 & 9.67 & 14.7 & 16.1 \\
\hline$T_{e 2} / \tau_{k}$ & 5.84 & 9.13 & 17 & 18 & 33.7 & 39.1 \\
\hline$\epsilon L_{f} / u^{\prime 3}$ & 1.09 & 0.72 & 0.60 & 0.54 & 0.43 & 0.41 \\
\hline
\end{tabular}

TABLE 1. Parameters and flow properties of the stationary homogenoeus isotropic turbulence (in arbitrary consistent units).

velocity field $\hat{u}_{i}$ in the wavenumber domain will be omitted in the inverse Fourier transform, which implies that the turbulent scales with size smaller than $2 \pi / k_{C H}$ will not be allowed to affect the particle motion. A second-order Runge-Kutta scheme is employed for the time advancement for both the flow field in the wavenumber space and the particle motion in the physical space. In order to avoid the error due to the time interpolation, we advance both the flow field and the particle motion with the same time step, which satisfies the CFL condition of the flow field and should be at least of one order less than the particle's relaxation time (see Yeh \& Lei 1991). As the instantaneous location of the particle $\boldsymbol{Y}(t)$ is not at the grid point of the flow field in general, the 13-point spatial interpolation method proposed by Yeung \& Pope (1988) is employed to evaluate $\boldsymbol{u}(\boldsymbol{Y}(t), t)$ from its neighbouring grid values in $(2 a)$. The triplex averaging procedure proposed by Wang \& Maxey (1993) is also employed for the particle calculation in order to reduce the statistical error. Thousands of particles were introduced uniformly into the flow field after the skewness of the velocity field had reached its asymptotic value. The initial velocity of a given particle is set equal to $\boldsymbol{v}_{d}$ plus the local fluid velocity. The particles were divided into six groups of different orientations for the body force. The six orientations, $\boldsymbol{g} /|\boldsymbol{g}|$, are $(1,0,0),(-1,0,0)$, $(0,1,0),(0,-1,0),(0,0,1)$ and $(0,0,-1)$. Statistical properties of the particle motion were calculated after time $T_{S}$ (defined later) when the average settling velocity of the particles had reached its asymptotic constant value. The triplex averaging procedure 
consists of three steps. First, the ensemble average is obtained among particles in each group of a specified orientation of $\boldsymbol{g}$. Next, the arithmetic average is taken among the six group of different orientations. Finally, the time average is calculated over the interval starting from $T_{S}$ to the end of the particle simulation $\left(T_{E}\right)$. Wang \& Maxey chose $T_{S}=2 T_{e 2}$ in their calculation, where $T_{e 2}$ is the eddy turnover time (defined in $\S 3)$.

\subsection{Numerical method using LES}

The method is the same as the method using DNS in the last section, except LES is employed to generate the flow field. The major difference between LES and DNS is that the high wavenumber components of the flow field (say, $k>k_{C}$ ) are not resolved in the LES, but the effect of the cutoff wavenumber components (for $k>k_{C}$ ) on the resolved low wavenumber components are modelled through the subgrid turbulent modelling. In the present study, the velocity and pressure fields in (1a) and $(1 b)$ are decomposed into a large-scale part and a subgrid-scale part,

$$
u_{i}(\boldsymbol{x}, t)=\overline{u_{i}}(\boldsymbol{x}, t)+u^{\prime \prime}(\boldsymbol{x}, t), \quad p(\boldsymbol{x}, t)=\bar{p}(\boldsymbol{x}, t)+p^{\prime \prime}(\boldsymbol{x}, t) .
$$

The large-scale fields (resolved fields), $\overline{u_{i}}$ and $\bar{p}$, are defined by mean of a convolution filter (see Ferziger 1983). The sharp-cutoff filter in Fourier space is employed in the present study. The equations governing the large-scale field are obtained by filtering $(1 a)$ and $(1 b)$. They are

$$
\begin{gathered}
\frac{\partial \overline{u_{i}}}{\partial x_{i}}=0 \\
\frac{\partial \overline{u_{i}}}{\partial t}+\frac{\partial}{\partial x_{j}}\left(\overline{\overline{u_{i}} \overline{u_{j}}}\right)=-\frac{\partial \bar{P}}{\partial x_{i}}-\frac{\partial \tau_{i j}}{\partial x_{j}}+v \frac{\partial^{2} \overline{u_{i}}}{\partial x_{j} \partial x_{j}}+f_{i},
\end{gathered}
$$

where

with

$$
\bar{P}=\bar{p} / \rho+\frac{1}{3} Q_{k k}, \quad \tau_{i j}=Q_{i j}-\frac{1}{3} Q_{k k} \delta_{i j},
$$

$$
Q_{i j}=\overline{\overline{u_{i}} u_{j}^{\prime \prime}}+\overline{\overline{u_{j}} u_{i}^{\prime \prime}}+\overline{u_{i}^{\prime \prime} u_{j}^{\prime \prime}} .
$$

The subgrid-scale Reynolds stress $\tau_{i j}$ is related to the large-scale field by using the eddy viscosity model :

$$
\tau_{i j}=2 v_{T} \overline{S_{i j}}=v_{T}\left(\frac{\partial \overline{u_{i}}}{\partial x_{j}}+\frac{\partial \overline{u_{j}}}{\partial x_{i}}\right),
$$

with the eddy viscosity $v_{T}$ represented by

$$
v_{T}=\left(C_{S} \Delta_{f}\right)^{2}\left(2 \overline{S_{i j} S_{i j}}\right)^{0.5} .
$$

Here the coefficient $C_{S}$ is taken from McMillan \& Ferziger (1979),

$$
C_{S}=0.128\left(1+\frac{24.5}{R_{s g s}}\right)^{-1}, \quad R_{s g s}=\frac{\left(\overline{S_{i j} S_{i j}}\right)^{0.5} \Delta_{f}^{2}}{v} .
$$

Note that the forcing term $f_{i}$ in $(6 b)$ is in general not affected by the filtering process, since the artifical forcing is applied to the low wavenumber components while the filtering process in LES is applied to the high wavenumber components. By comparing $(6 a)$ and $(6 b)$ for LES with $(1 a)$ and $(1 b)$ for DNS, we found that the equations are of the same form for both methods if $\overline{u_{i}}, \bar{P}$ and $\overline{\bar{u}_{i} \bar{u}_{j}}+\tau_{i j}$ in LES are replaced by $u_{i}$, $p / \rho$ and $u_{i} u_{j}$ in DNS. The numerical procedures for calculating the flow field are thus 
similar to those in the last section, with the subgrid Reynolds stress term calculated in a way similar to the viscous term. Details of the calculation of the particle motion are also the same as those in $\S 2.2$ provided $u_{i}$ in $(2 a)$ is replaced by $\overline{u_{i}}$.

\section{Results in stationary turbulence}

\subsection{Flow characteristics}

As discussed in Ferziger (1983), the DNS/LES calculation has three stages: the initial (developing) period, the mature period, and the final period when the assumptions in the calculation are violated. The end of the initial period is characterized when the skewness reaches its asymptotic value (around -0.4 to -0.5 ) and $k_{\max } \eta \geqslant 1$. The mature period is the time range we employ to study the turbulence characterestics. We start our particle calculation at the beginning of the mature period, evaluate the particle's statistical properties after the effect of the artifical initial conditions is negligible and the average settling velocity of particles reaches its asymptotic constant value (see §3.2), and terminate the particle calculation at the end of the mature period.

Six cases were simulated in the present study, and were employed to study the particle statistics. Table 1 lists the parameters for the simulation and the flow properties of the mature period (in arbitrary consistent units). The parameters for the simulation include the size of the calculation domain $\left(L^{3}\right)$, the number of grids $\left(N^{3}\right)$, the method for generating the flow field, the forcing amplitude $\left(S_{f c}\right)$ and the forcing time scale $\left(T_{f c}\right)$ associated with the artifical forcing term, the initial root mean square of the velocity fluctation $\left(u_{f 0}^{\prime}\right)$ and the inital integral length scale $\left(L_{f 0}\right)$ for starting the calculations in (4), and the kinematic viscosity $(v)$. Note that cases $C$ and D are similar except they are simulated using different methods (and hence different grids). The flow properties of the mature period include the root mean square of the velocity fluctuation $\left(u^{\prime}\right)$, the integral length scale $\left(L_{f}\right)$, the dissipation $(\epsilon)$, the skewness $(S)$, the eddy turnover time scales $\left(T_{e 1}\right.$ and $\left.T_{e 2}\right)$, the Kolmogorov length scale $(\eta)$, the Kolmogorov timescale $\left(\tau_{k}\right)$, the Kolmogorov velocity scale $\left(v_{k}\right)$, the Taylor microscale for length $(\lambda)$, the Reynolds number based on $\lambda\left(R_{\lambda}\right)$, and the greatest dimensionless wavenumber simulated in the calculation $\left(k_{\max } \eta\right)$. Here

$$
u^{\prime}=\left(\overline{u^{2}}\right)^{1 / 2}, \quad L_{f}=\frac{1}{3}\left(L_{f 1}+L_{f 2}+L_{f 3}\right), \quad \epsilon=2 v \int_{0}^{\infty} k^{2} E(k) \mathrm{d} k,
$$

and

$$
S=\frac{\overline{\left(\partial u_{1} / \partial x_{1}\right)^{3}}}{\left[\overline{\left(\partial u_{1} / \partial x_{1}\right)^{2}}\right]^{3 / 2}}
$$

where the integral length scale is defined through

$$
\overline{u_{i}^{2}} L_{f i}=\int_{0}^{\infty} u_{i}(r) u_{i}(0) \mathrm{d} r, \quad i=1,2,3,
$$

the energy spectrum $E(k)$ is defined as

$$
\frac{3}{2} u^{\prime 2}=\int_{0}^{\infty} E(k) \mathrm{d} k
$$

and $\overline{(\cdot)}$ denotes the average of the quantity $(\cdot)$ evaluated from the values at the grids. With $u^{\prime}, L_{f}, \epsilon$ and $S$ calculated from the numercial results of the flow field, one can 
evaluate

$$
\begin{gathered}
T_{e 1}=\frac{L_{f}}{u^{\prime}}, \quad T_{e 2}=u^{\prime 2} / \epsilon \\
\eta=\left(\frac{v^{3}}{\epsilon}\right)^{1 / 4}, \quad \tau_{k}=\left(\frac{v}{\epsilon}\right)^{1 / 2}, \quad v_{k}=(v \epsilon)^{1 / 4}, \\
\lambda=\left(\frac{15 v}{\epsilon}\right)^{1 / 2} u^{\prime}=15^{1 / 2} \tau_{k} u^{\prime},
\end{gathered}
$$

and

$$
R_{\lambda}=\frac{u^{\prime} \lambda}{v} .
$$

Note that $T_{e 2}$ is the eddy turnover time scale employed in Wang \& Maxey (1993). Also listed in table 1 are the dimensionless quantities $u^{\prime} / v_{k}, L_{f} / \eta, L_{f} / \lambda, T_{e 1} / \tau_{k}, T_{e 2} / \tau_{k}$ and $\epsilon L_{f} / u^{\prime 3}$. These quantites possess monotonically varying behaviour as $R_{\lambda}$ increases as expected if either case C or D is omitted from the list. Cases C and D have similar values of $R_{\lambda}$, but are calculated using different methods. Table 1 shows that the results for case $C$ and $D$ are similar, which indicates the validity of the present LES. We have tried different values of $S_{f c}$ and $T_{f c}$ during the calculations, and checked the asymptotically stationary property of the time evolution of $R_{\lambda}, u^{\prime}$ and $S$. Note that the values of the skewness for all cases in table 1 have reached the theoretical asymptotic value.

Figure 1 shows the energy and the dissipation spectra for the simulated cases in the present study. Note that the vorticity spectrum is related to the dissipation spectrum through a factor $2 v$. In order to check our calculations, the theoretical result based on Townsend's (1951) model and the calculation for $R_{\lambda} \approx 150$ according to Vincent $\&$ Meneguzzi (1991) are also plotted in figure 1. Note that the definition for $R_{\lambda}$ in Vincent \& Meneguzzi is slightly different from that in the present study. The present results for the dissipation spectra agree asymptotically with Townsend's model for large $k \eta$, and the results for large $R_{\lambda}$ (based on LES) agree qualitatively with the DNS calculation by Vincent $\&$ Meneguzzi. The results at low wavenumber for large $R_{\lambda}$ are quantitatively different from the result by Vincent \& Meneguzzi, which is expected because different forcing schemes were applied for different calculations. Also note that the results for large $R_{\lambda}$ show a short inertial subrange with slope $-5 / 3$ like that in Vincent $\&$ Meneguzzi. The case for $R_{\lambda} \approx 65$ has been simulated by both the DNS (case C, $96^{3}$ grids, $R_{\lambda}=65.3$ ) and the LES (case D, $32^{3}$ grids, $R_{\lambda}=69.8$ ) in the present study, and the results from both methods in figure 1 agree nicely with each other. Detailed examination of the data shows that the LES resolves approximately $95 \%$ of the total energy and $75 \%$ of the total dissipation of the results using DNS. The wavenumber corresponding to the peak value of the dissipation spectrum (or the eddies with maximum vorticity), $k_{\omega}$, decreases and tends to approach a constant value as $R_{\lambda}$ increases. The values for $k_{\omega} \eta$ are $0.172,0.134$ and 0.122 for $R_{\lambda}$ equal to $65.3,133$ and 153 , respectively, based on the present calculations. The data for $R_{\lambda}=22.6$ and 35.7 in figure $1(b)$ show some fluctuations at low wavenumbers, which is due to the forcing associated with the simulation of stationary turbulence. The corresponding results for decaying homogeneous isotropic turbulence are smooth (see Yang 1997), and $k_{\omega} \eta=0.26$ and 0.23 for $R_{\omega}=22.6$ and 35.7, respectively. 

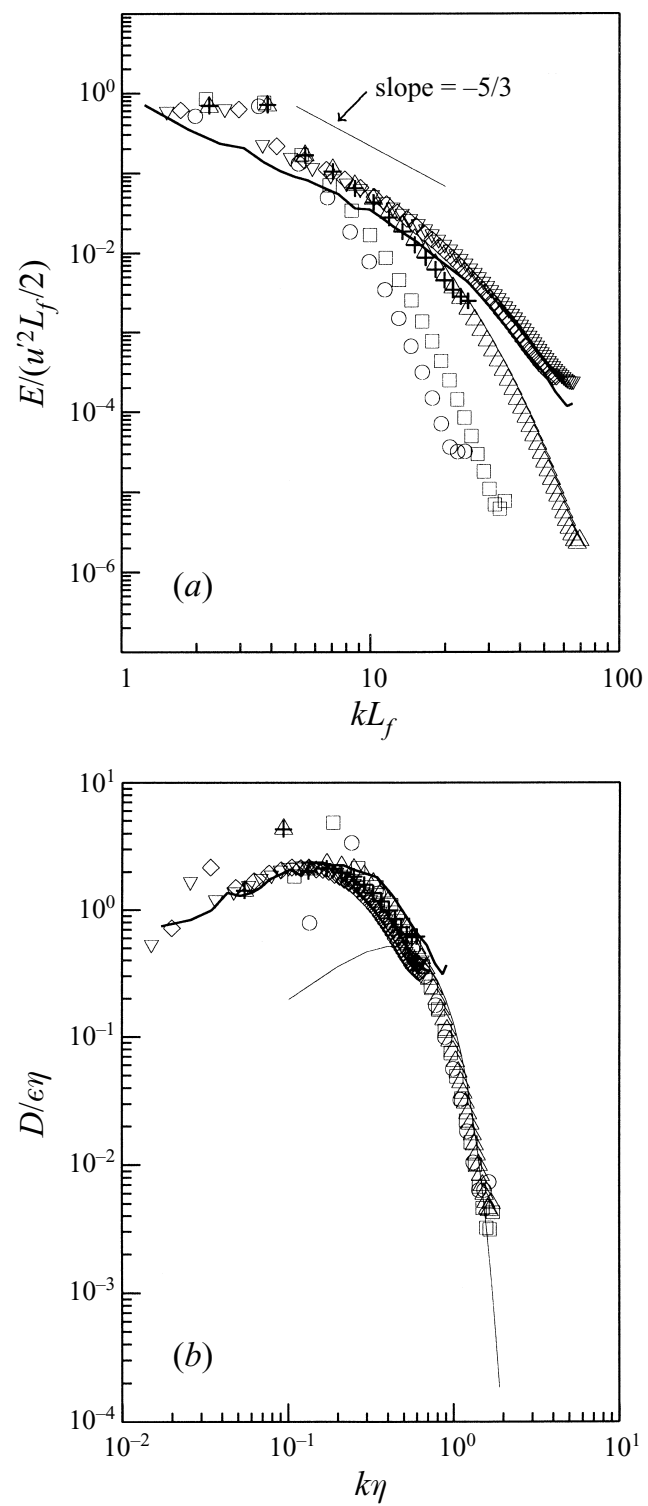

FIGURE 1. (a) The energy, and $(b)$ the dissipation spectra for the flow fields. $O, R_{\lambda}=22.6 ; \square$, $R_{\lambda}=35.7 ; \triangle, R_{\lambda}=65.3$ (DNS, $96^{3}$ grids);,$+ R_{\lambda}=69.8$ (LES, $32^{3}$ grids); $\diamond, R_{\lambda}=133$ and $\nabla$, $R_{\lambda}=153$. The numerical result by Vincent \& Meneguzzi (1991) is represented by the thick curve $\left(R_{\lambda} \approx 150\right.$, DNS, $240^{3}$ grids). The thin curve in $(b)$ is the theoretical result based on Townsend's model (1951).

\subsection{The transient time scale for the particle motion}

According to Riley \& Patterson (1974), the initial stage of the particle calculation is affected by the imposed initial conditions, and they proposed that statistics of the particle motion which are free of initial conditions can be taken after the relative velocity between the particle and the fluid has reached the maximum value. Figure 2 (a) shows the time evolution of the ensemble average of the root mean square of the relative velocity component perpendicular to $\boldsymbol{v}_{d}$ after the first two steps of the triplex 

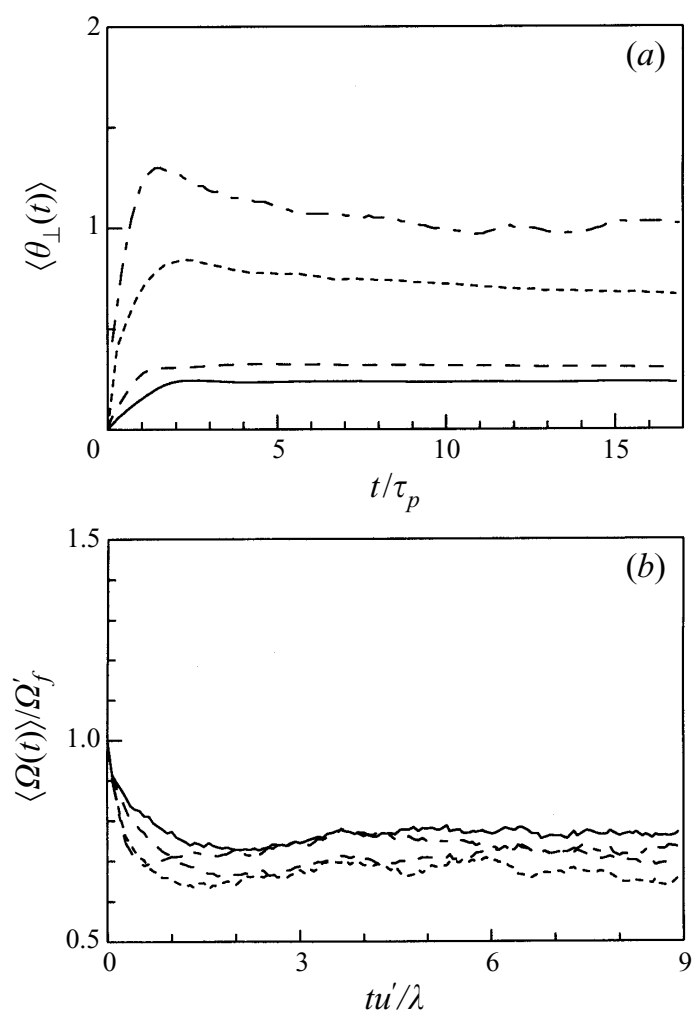

FIGURE 2. Evolution of the ensemble average of $(a)$ the root mean square of the relative velocity component, and $(b)$ the dimensionless enstrophy for $R_{\lambda}=65.3$ and $v_{d} / v_{k}=1 .-, \tau_{p} / \tau_{k}=0.15$; $-—-, \tau_{p} / \tau_{k}=0.25 ;-\cdots, \tau_{p} / \tau_{k}=1$ and,$--- \tau_{p} / \tau_{k}=2.5$.

averaging procedures, $\left(\left\langle\theta_{\perp}(t)\right\rangle\right)$. It is found that the time scale required for $\left\langle\theta_{\perp}(t)\right\rangle$ to reach the asymptotic stationary value is of order $\tau_{p}$, the particle's relaxation time. However, the time scale for evaluating the ensemble average of the settling velocity deserves further consideration. As mentioned in $\S 1$, the increase of the average settling velocity is a consequence of the non-uniform particle distribution due to the highly intermittent vortical structure. Thus knowledge of the transient time scale for the particle accumulation is important for the correct estimation of the increase of the average settling velocity. As we know from $\S 1$ that the particles tend to accumulate in the region of low vorticity, a good indicator for the accumulation of particles is the value of the mean enstrophy (square of the vorticity) where the particle locates. Figure 2(b) shows the time evolution of $\langle\Omega(t)\rangle / \Omega_{f}^{\prime}$ for different particle parameters. Here $\langle\Omega(t)\rangle$ is the mean enstrophy at the instantaneous locations of the particles after the first two steps of the triplex averaging procedures, and $\Omega_{f}^{\prime}$ is the mean enstrophy of the flow field evaluated at the grids, which is constant for stationary homogeneous isotropic turbulence. In the absense of particle inertia, there is no particle accumulation, and $\langle\Omega(t)\rangle / \Omega_{f}^{\prime}$ approaches unity at large time. For particles with a given finite value of inertia, the particles tend to accumulate in the region of low vorticity according to Wang \& Maxey (1993), and $\langle\Omega(t)\rangle / \Omega_{f}^{\prime}$ should approach an asymptotic constant value less than unity at large time. Figure $2(b)$ indeed shows such a result, and the time scale for reaching the approximate constant value is of order $\lambda / u^{\prime}\left(=\sqrt{15} \tau_{k}\right)$, which is a time scale related to the flow but not the particles. This 

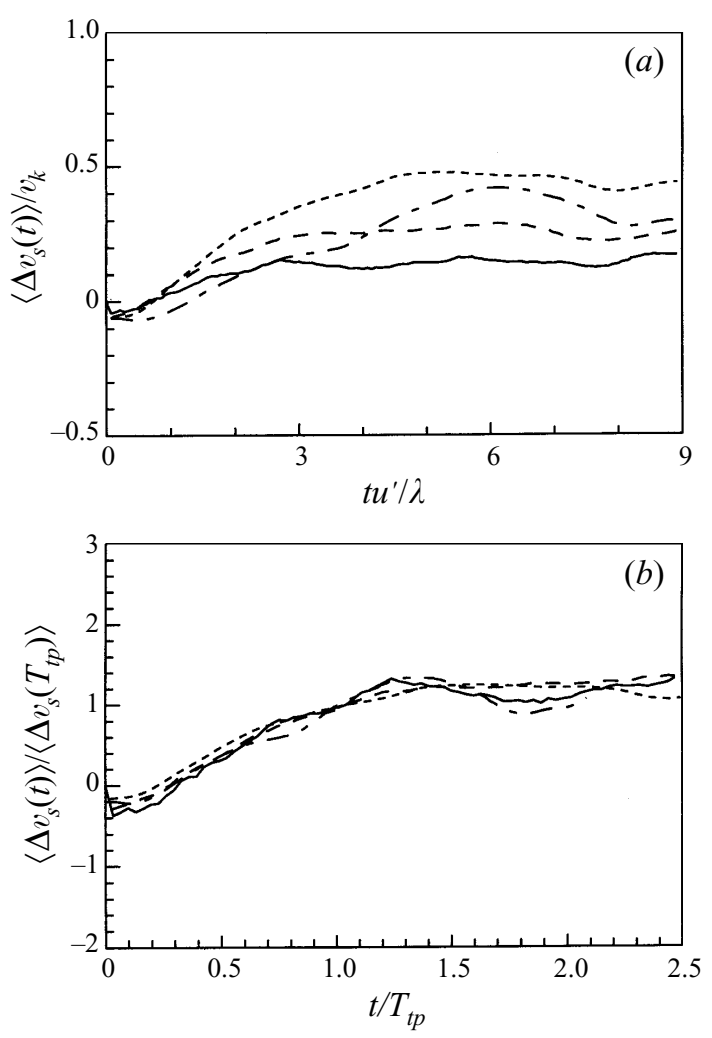

FIGURE 3. Evolution of $\left\langle\Delta v_{S}(t)\right\rangle$ using $(a) \lambda / u^{\prime}$ and $(b) T_{t p}$ as time scales. The curves are the same as those in figure 2 . Here $T_{t p}=2 \lambda / u^{\prime}+5 \tau_{p}$.

can be understood as follows. As the spacing between the tubular vortices ('worms') is of order $\lambda$ according to Vincent $\&$ Meneguzzi (1991), and the velocity scale for the particle is of order $u^{\prime}$, the velocity scale of the flow in the low vorticity region, it follows that the average time for a particle to reach the low vorticity region is of order $\lambda / u^{\prime}$. According to figure 2 , the transient time scale for estimating the increase of the settling velocity is thus related to both the time scale of the particle, $\tau_{p}$, and the time scale of the flow, $\lambda / u^{\prime}$. Figure 3(a) shows $\left\langle\Delta v_{S}(t)\right\rangle$, the magnitude of the ensemble average of the settling velocity after the first two steps of the triplex averaging procedures, and we found that there are different transient response time for different particle inertia. However, if we plot the variation of $\left\langle\Delta v_{S}(t)\right\rangle$ with a dimensionless time normalized by $2 \lambda / u^{\prime}+5 \tau_{p}$ in figure $3(b)$, we find that the results for different particle inertia approach an essentially constant value when $t \geqslant T_{t p}=2 \lambda / u^{\prime}+5 \tau_{p}$. Although the above results in figures 2 and 3 are for $R_{\lambda}=65.3$, the calculations for other $R_{\lambda}$ not shown in this paper also confirm such findings. Thus $T_{t p}$ seems to be the appropriate time scale associated with the transient behaviour of $\left\langle\Delta v_{S}(t)\right\rangle$ at least for the ranges of parameters in the present study. The particle statistics is evaluated only when $t \geqslant T_{t p}$ during the particle simulation. Wang \& Maxey (1993) proposed to take the ensemble average of the settling velocity twice the eddy turnover time $\left(2 T_{e 2}\right)$ after the start of the particle calculations based on their simulations. Recall that the increase of the ensemble average of the settling velocity is maximized when $\tau_{p} \approx \tau_{k}$ according to Wang \& Maxey. On taking $\tau_{p}=\tau_{k}$, It is found that $2 T_{e 2} / T_{t p}=0.91,1.42,2.65,5.33$ 

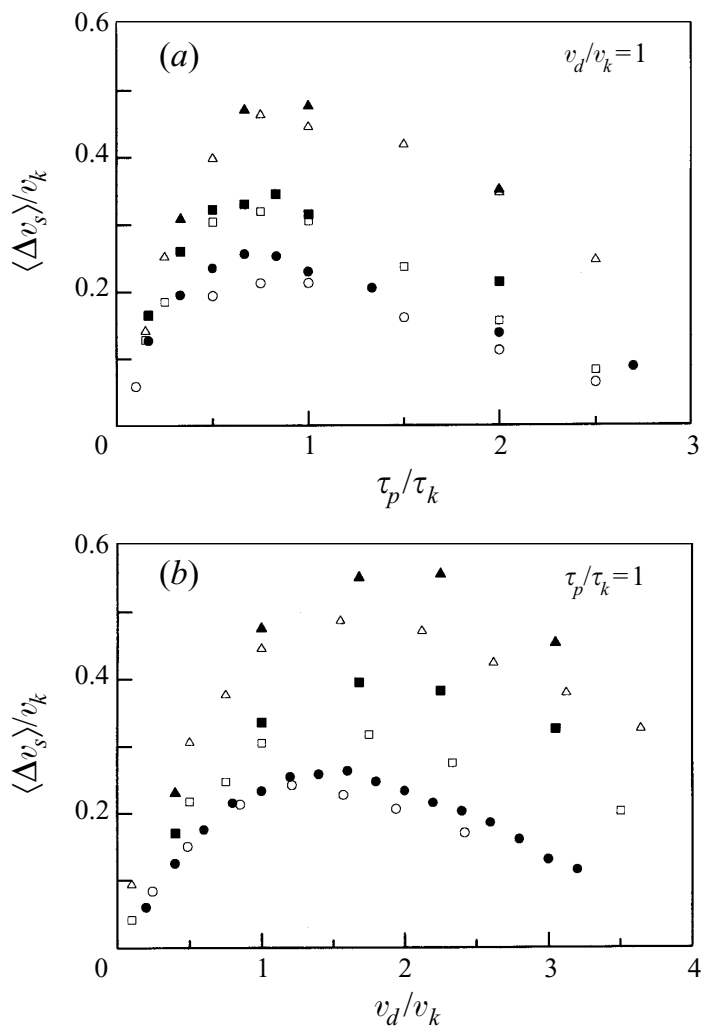

FIGURE 4. The increase of the average settling velocity for different parameters. Present results: $O$, $\square$ and $\triangle$ for $R_{\lambda}=22.6,35.7$ and 65.3, respectively. Results from Wang \& Maxey: $R_{\lambda}=21.2,31$ and 61.5 , respectively.

and 6.18 for cases A, B, C, E and F, respectively, according to table 1. In the present study, $T_{S}=T_{t p}$ is employed for the last stage of the triplex averaging procedures (see $\S 2.2)$.

\subsection{The average settling velocity of particles}

The present particle results are validated by comparing the increase of the average settling velocity after the triplex averaging procedures, $\left\langle\Delta v_{S}\right\rangle$, with the calculations by Wang \& Maxey (1993). The number of particles for evaluating the particle statistics is $3072 \times 6$ (recall that there are 6 orientations of the body force field for the second step of the triplex averaging procedure). There are two particle parameters for a given flow field, $\tau_{p}$ and $v_{d}\left(=g \tau_{p} / C_{0}\right)$, which are related to the particle inertia and the strength of the body force field, respectively. Figure 4 shows $\left\langle\Delta v_{S}\right\rangle$ for different values of $\tau_{p}$ and $v_{d}$. The present results agree nicely with the calculations by Wang \& Maxey, except for some results with large values of $v_{d}$. The discrepancy is due to the fact that $R_{p}$ is sufficiently large such that the results based on the Stokes drag law $(C=1$ in $(2 a)$ ) in Wang \& Maxey are not accurate for large $v_{d}$. The qualitative behaviour that the results obtained by using the nonlinear drag law are less than those obtained by using the linear Stokes drag law is consistent with the previous finding according to Wang \& Maxey (1993).

Consider the problem in a given flow field (given $R_{\lambda}$ ). For a fixed value of $v_{d}$, $\left\langle\Delta v_{S}\right\rangle$ is zero when $\tau_{p}=0$ since there is no particle accumulation. As $\tau_{p}$ increases, 

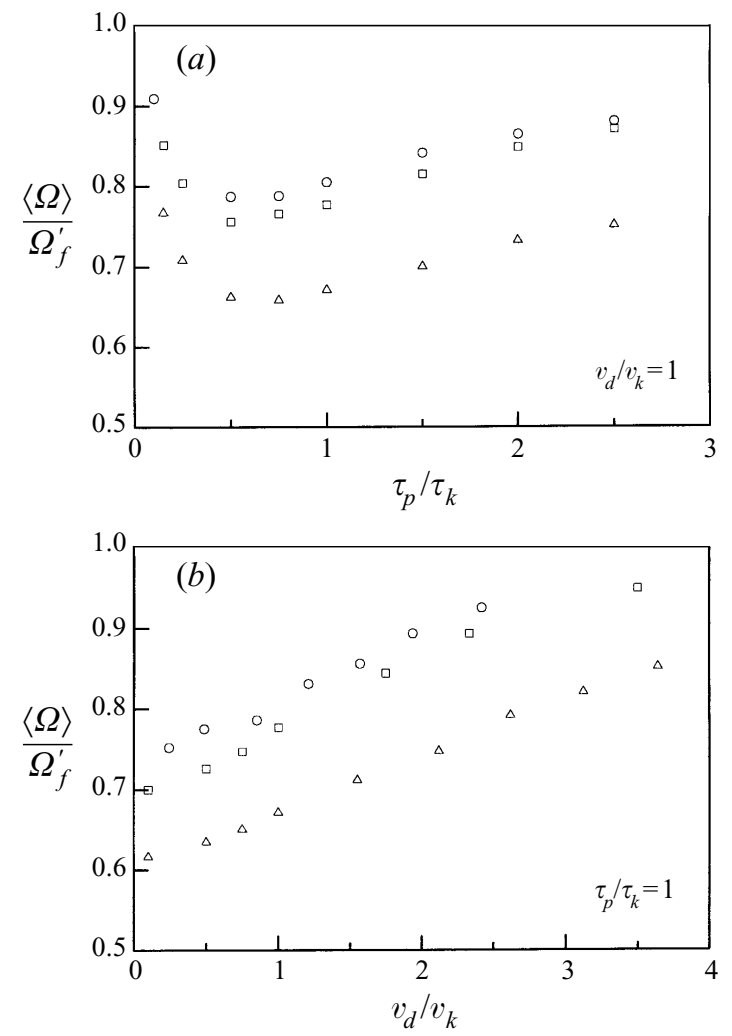

Figure 5. The ensemble average of the dimensionless enstrophy at the locations of the particles for different parameters. The symbols are the same as those in figure 4 .

the centrifugal effect increases and drives more particles toward the region between vortices (low vorticity region). This effect of particle accumulation together with the preferential sweeping mechanism make the value of $\left\langle\Delta v_{S}\right\rangle$ increase as $\tau_{p}$ increases. However, when $\tau_{p}$ reaches a certain finite value and increases further, the relative importance of the 'sideways' centrifugal effect and the 'downward' body force effect decreases. It follows that the degree of particle accumulation, and thus $\left\langle\Delta v_{S}\right\rangle$, decrease as $\tau_{p}$ increases. Finally, when $\tau_{p}$ is sufficiently greater than any integral time scale of the flow, the turbulent fluid velocity seen by the particle appears as uncorrelated random noise and $\left\langle\Delta v_{S}\right\rangle$ drops to zero. Figure $4(a)$ indicates that $\left\langle\Delta v_{S}\right\rangle$ is maximized at $\tau_{p} / \tau_{k} \approx 0.5-1.0$ for given values of $v_{d} / v_{k}$ and $R_{\lambda}$, and $\left\langle\Delta v_{S}\right\rangle$ increases as $R_{\lambda}$ increases. The phenomenon of particle accumulation is illustrated in figure $5(a)$, which shows the variations of $\langle\Omega\rangle / \Omega_{f}^{\prime}$ with $\tau_{p} / \tau_{k}$ at $v_{d} / v_{k}=1$ for different values of $R_{\lambda}$. Here $\langle\Omega\rangle$ is the ensemble average of the enstrophy at the locations of the particles based on the triplex averaging procedures. It is found that the effect of particle accumulation in the low vorticity region is also maximized at $\tau_{p} / \tau_{k} \approx 0.5-1.0$. By comparing the detailed results of figures $4(a)$ and $5(a)$, it is found that for a given value of $v_{d}$, the increase of the settling velocity is consistently proportional to the degree of particle accumulation. Figure 4(b) shows that for given values of $R_{\lambda}$ and $\tau_{p} / \tau_{k}$, there also exists a maximum value of $\left\langle\Delta v_{S}\right\rangle$ at a finite value of $v_{d}$. However, the location of the maximum value of $\left\langle\Delta v_{S}\right\rangle / v_{k}$ shifts continuously rightward as $R_{\lambda}$ increases, which implies that $v_{k}$ may not be an appropriate velocity scale for the present problem, which will be discussed later 

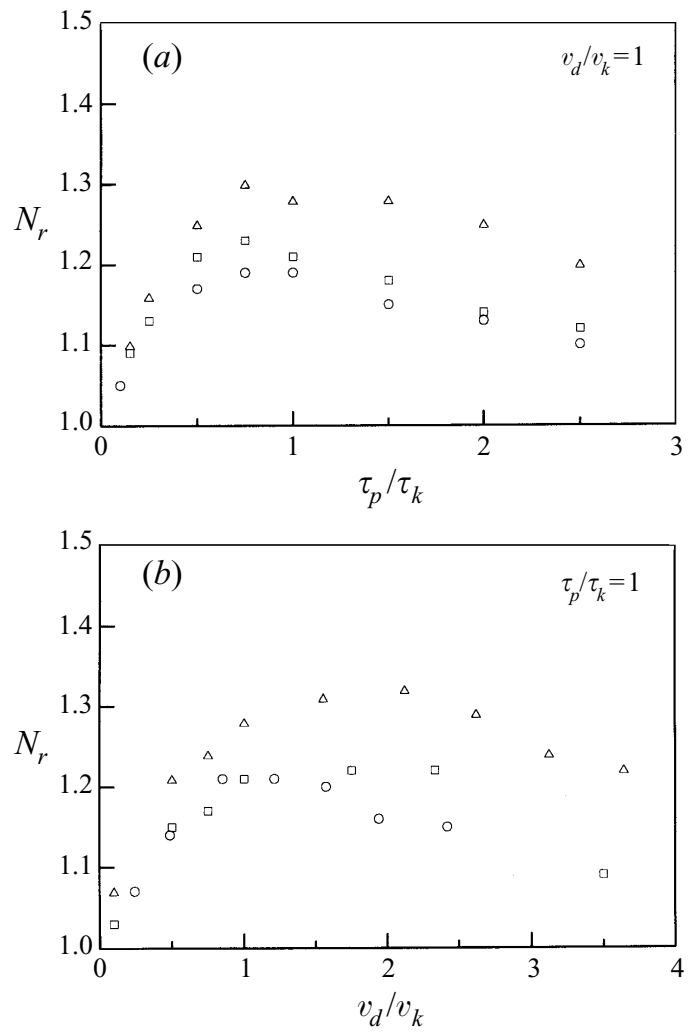

FIGURE 6. The parameter for illustrating the preferential sweeping phenomenon, $N_{r}$, for different parameters. The symbols are the same as those in figure 4

in $\S 3.6$. The phenomenon of particle accumulation corresponding to figure $4(b)$ is shown in figure $5(b)$. It is found that the effect of particle accumulation is maximized when $v_{d} \approx 0$, and vanishes as $v_{d}$ increases. Unlike the cases for fixed $v_{d}$ in figures $4(a)$ and $5(a)$, the variation of $\left\langle\Delta v_{S}\right\rangle$ for fixed $\tau_{p}$ in figure $4(b)$ is not directly related to the degree of particle accumulation in figure $5(b)$. The reason is that the accumulation of particles in the low vorticity region is only a necessary condition for the increase of the average settling velocity. It is also required that more particles are accumulated in the 'downward sweeping' region in comparison with those in the 'upward sweeping' region (the preferential sweeping phenomenon according to Wang \& Maxey 1993). Figure 6 is employed for quantifying the preferential sweeping phenomenon. Let $\boldsymbol{u}$ be the local fluid velocity at the instantaneous location of the particle. Define $N_{r}$ as the ratio of the number of particles with $\boldsymbol{u} \cdot \boldsymbol{v}_{d}>0$ ('downward sweeping') to those with $\boldsymbol{u} \cdot \boldsymbol{v}_{d}<0$ ('upward sweeping'). Figure 6 shows that $N_{r} \geqslant 1$ for all cases, which implies that more particles indeed stay in the 'downward sweeping' region. For $v_{d} / v_{k}=1$, the variation of $N_{r}$ with parameters in figure $6(a)$ is consistent with the results in figures $4(a)$ and $5(a)$, and $N_{r}$ is also maximized at $\tau_{p} / \tau_{k} \approx 0.5-1$ for different parameters. For $\tau_{p} / \tau_{k}=1$, figure $6(b)$ shows that the preferential sweeping is weak when $v_{d} / v_{k}$ is small. In fact, there is no preferential direction, and thus no preferential sweeping, when $v_{d}=0$ (zero body force). It follows that although we have a large degree of particle accumulation in figure $5(b)$ near $v_{d}=0,\left\langle\Delta v_{S}\right\rangle$ is not maximized at $v_{d}=0$ but at another finite value as shown in figure $4(b)$. By comparing figure $4(b)$ with figure 

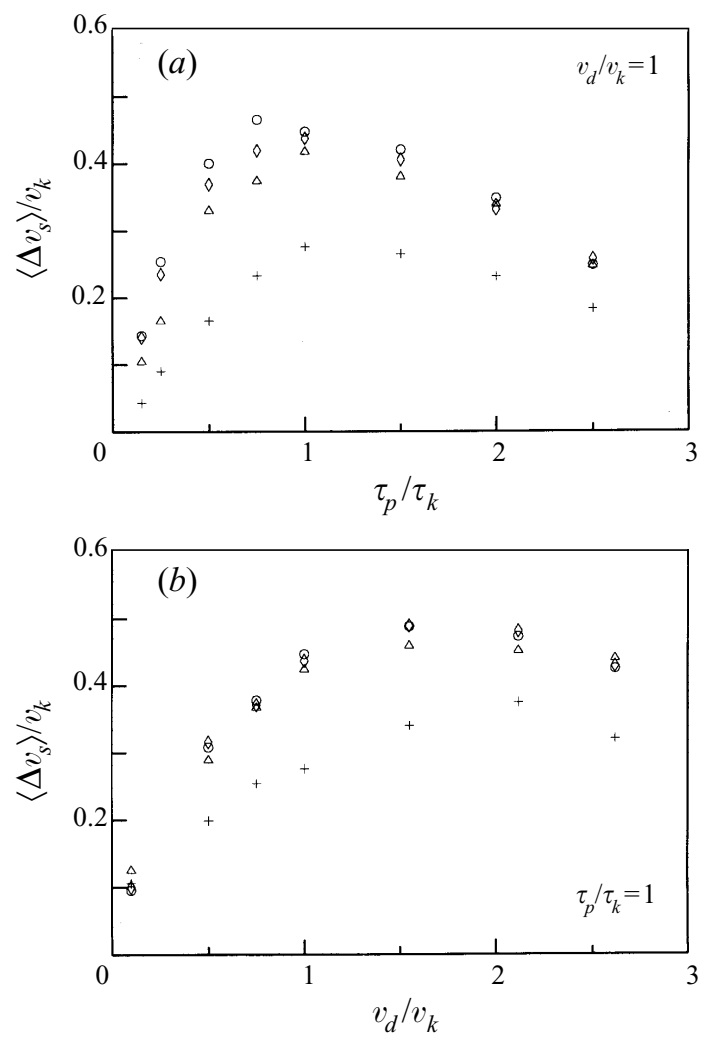

FIGURE 7. Effect of small turbulent scales on $\left\langle\Delta v_{S}\right\rangle$ in stationary turbulence at $R_{\lambda}=65.3$.

O, all simulated modes; $\diamond, k_{C H} \eta=0.5 ; \triangle, k_{C H} \eta=0.4$; and,$+ k_{C H} \eta=0.2$.

$6(b)$, the locations of the maximum value of $\left\langle\Delta v_{S}\right\rangle / v_{k}$ are consistent with those of $N_{r}$. Also note that when $v_{d}$ is sufficiently large (large 'crossing trajectory effect') for a fixed value of $\tau_{p}$, the duration of a particle in an 'eddy' is so short that the effect of turbulence on the particle accumulation is negligible, and $\left\langle\Delta v_{S}\right\rangle$ drops to zero.

\subsection{Effect of the small scales}

In order to study the effect of small eddies, we set $k_{C L}=k_{\min }$ and $k_{C H}<k_{\max }$. Thus different ranges of the high wavenumber components $\left(k_{C H}<k<k_{\max }\right)$ can be omitted by setting different values of $k_{C H}$. Recall that the values of $k_{\max } \eta$ for different cases are listed in table 1 . Figures 7 and 8 show the results of the average settling velocity and the particle accumulation for different values of $\tau_{p} / \tau_{k}, v_{d} / v_{k}$ and $k_{C H} \eta$ at $R_{\lambda}=65.3$. The agreement between the results using all turbulent scales $\left(k_{\max } \eta=1.71\right.$, all simulated modes) and those using only partial turbulent scales $\left(k_{C H} \eta=0.5\right.$, excluding the high wavenumber modes from 0.5 to 1.71 ) indicates that the high wavenumber modes have essentially no effect on the particle accumulation and the particle's settling velocity. However, the results with $k_{C H} \eta=0.2$ are substantially less than the values calculated in the flow field with all the simulated modes. The results for $R_{\lambda}=22.6$ and 35.7 (not shown here) are similar to those for $R_{\lambda}=65.3$. The cutoff wavenumber in the LES by Yeh \& Lei (1991) for $R_{\lambda} \approx 44$ is roughly $0.2 / \eta-0.4 / \eta$, the vorticity structure and hence the phenomenon of particle accumulation were not simulated correctly, and thus the results of the average settling velocity in Yeh \& Lei 

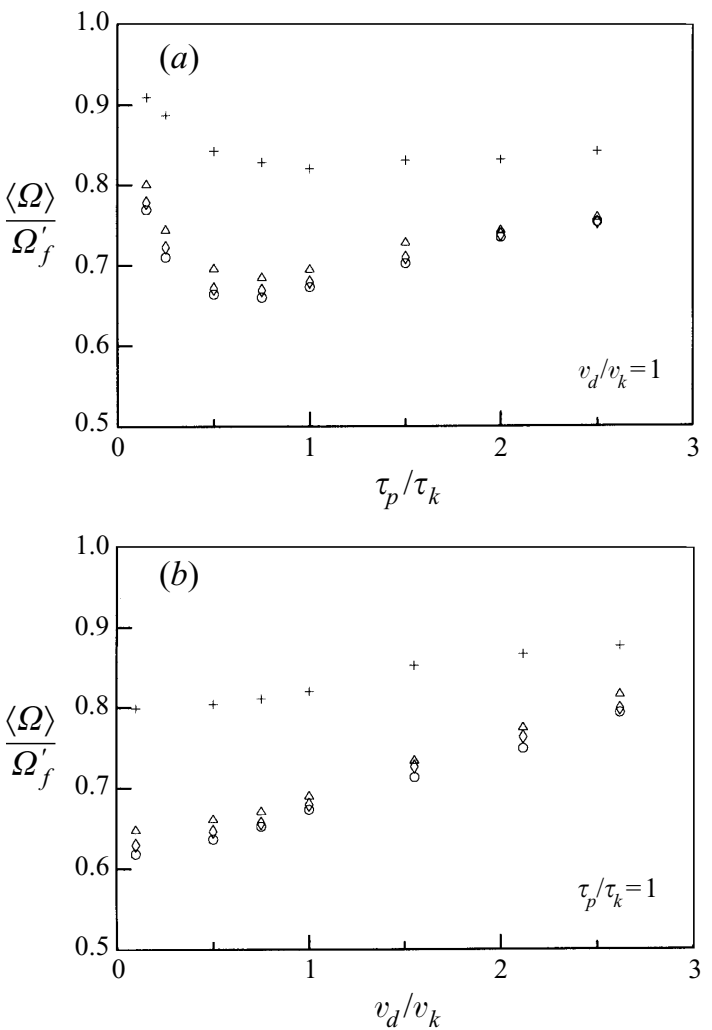

FIGURE 8. Effect of small turbulent scales on the average dimensionless enstrophy evaluated at the locations of the particles at $R_{\lambda}=65.3$. The symbols are the same as those in figure 7 .

were underestimated. Note that the 'traditional' LES employed by Yeh \& Lei is still applicable for studying the particle dispersion problem.

As in Wang \& Maxey (1993), the instantaneous results within a thin slice of the flow field (with thickness equal to the grid spacing) are employed for illustrating the physics. The particle velocity vectors within the volume of the slice are projected onto the centreplane of the slice. The background plots of figures $9(a), 9(c)$ and $9(e)$ are the instantaneous dimensionless enstrophy contours at the centreplane. The corresponding distributions of the instantaneous fluid velocity are shown in figures $9(b), 9(d)$ and $9(f)$. The maximum values in figures $9(a), 9(c)$ and $9(e)$ are $37.12,18.39$ and 2.90 , respectively. The phenomenon of particle accumulation in the low vorticity (enstrophy) region can be observed clearly in figure $9(a)$, i.e. most of the starting points of the particle velocity vectors are at the low vorticity region. The preferential sweeping phenomenon is illustrated through the consistence of the particle velocity vectors in figure $9(a)$ and the fluid velocity vectors in figure $9(b)$.

Consider the results using all simulated modes in figures $9(a)$ and $9(b)$ and the results for $k_{C H} \eta=0.6$ in figures $9(c)$ and $9(d)$. Although the detailed values in the high enstrophy regions are different, the essential structure of the enstrophy contours in figures $9(a)$ and $9(c)$ are similar. Also the velocity fields in figure $9(b)$ and $9(d)$ are almost the same. The discrepancy for enstrophy at the high vorticity regions is thought to contribute negligibly to the average settling velocity since particles are rare at the high vorticity regions. Detailed examination of the data shows that the case 


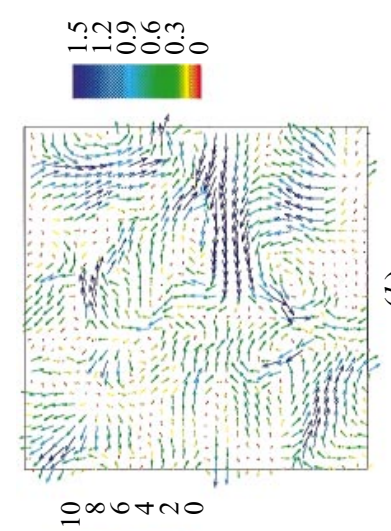

a
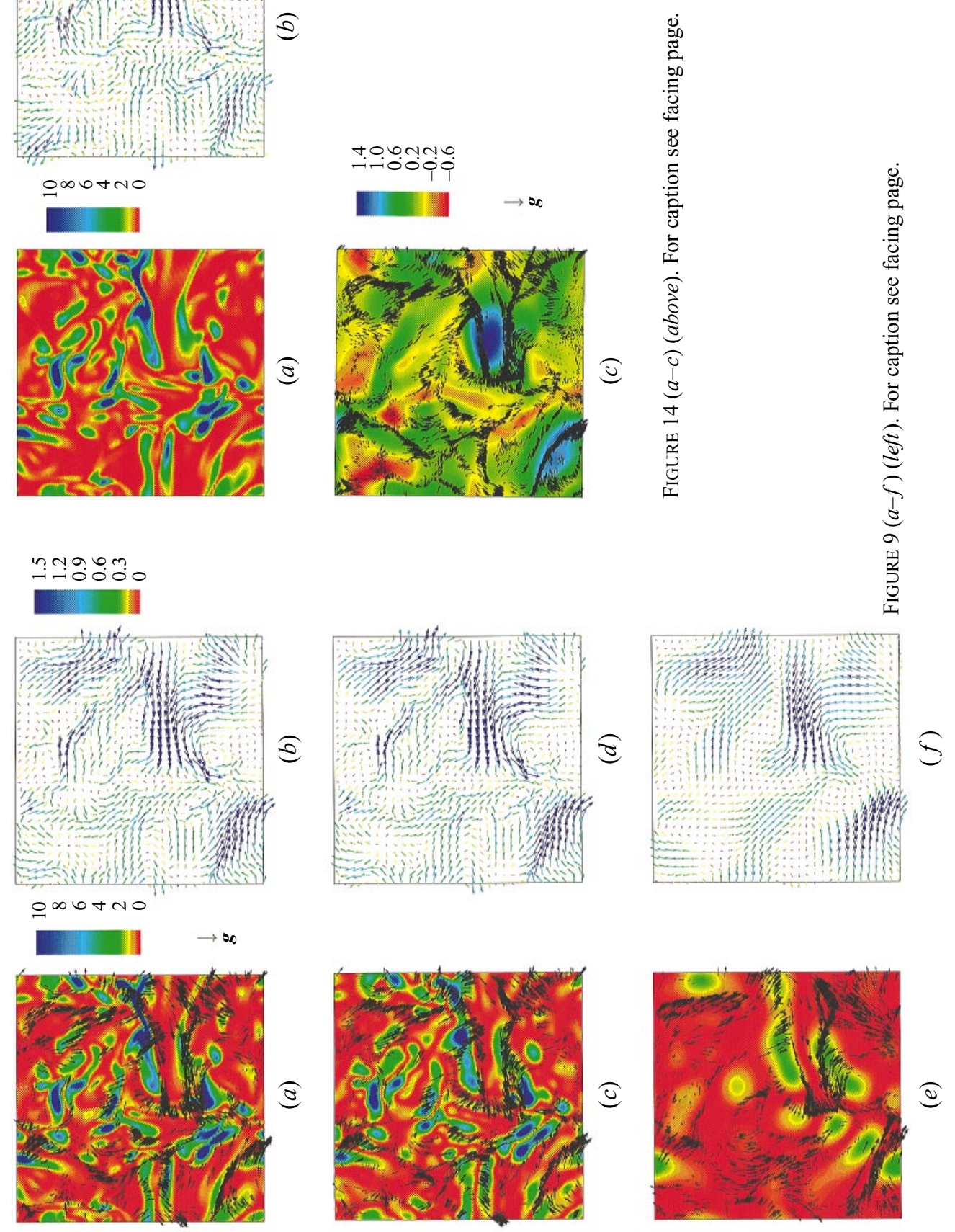

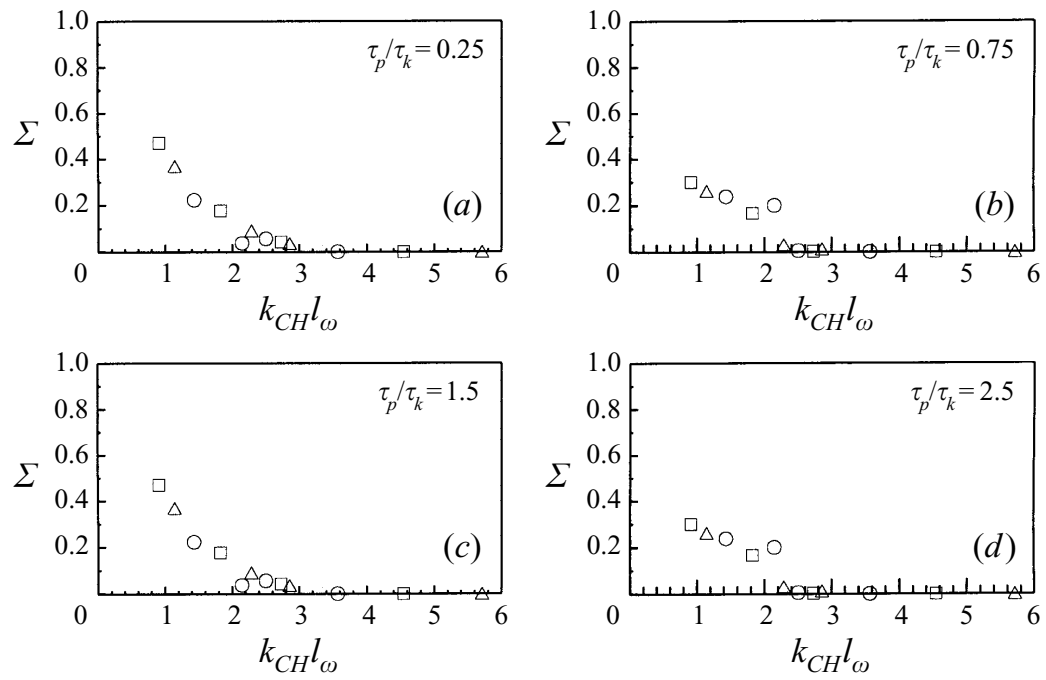

FIGURE 10. The relative error of the increase of the average settling velocity, $\Sigma$, for different values of $\tau_{p} / \tau_{k}, k_{C H} l_{\omega}$ and $R_{\lambda}$ at $v_{d} / v_{k}=1$. The symbols are the same as those in figure 4 .

with $k_{C H} \eta=0.6$ resolves approximately $75 \%$ of the total enstrophy and $98 \%$ of the total energy of the case with all simulated modes. It follows that the sites of particle accumulation and the average settling velocity for the case with all simulated modes are basically the same as those with $k_{C H} \eta=0.6$. On the other hand, although the flow field with $k_{C H} \eta=0.2$ in figure $9(f)$ still possesses some essential similar features as in figure $9(b)$ (resolving $75 \%$ of the total energy), the enstrophy distribution for $k_{C H} \eta=0.2$ (figure $9 e$ ) is completely different from that in figure 9(a) (resolving only $25 \%$ of the total enstrophy). It follows that the particle accumulation, and thus the average settling velocity for case with $k_{C H} \eta=0.2$ are different from those for the case with all simulated modes.

Denote the value of $\left\langle\Delta v_{S}\right\rangle$ for the case with all simulated modes by $A$, and the value of $\left\langle\Delta v_{S}\right\rangle$ for a given $k_{C H}$ by $A^{*}$. Define the relative error $\Sigma$ as

$$
\Sigma=\frac{A-A^{*}}{A}
$$

which depends on $R_{\lambda}, \tau_{p} / \tau_{k}$, and $v_{d} / v_{k}$. Figure 10 plots the values of $\Sigma$ for some of our typical simulated results using $l_{\omega}^{-1}$ as the scale for $k_{C H}$. Recall that $l_{\omega}=1 / k_{\omega}$, with $k_{\omega}$ the wavenumber corresponding to the maximum of the dissipation (or vorticity) spectrum. The results in figure 10 together with other results not shown in the present paper suggest that the relative error is negligible for $k_{C H} l_{\omega}>2.5$. It follows that LES is still applicable for generating the flow field for the simulation of $\left\langle\Delta v_{S}\right\rangle$ provided

FIGURE 9. $(a),(c)$ and $(e)$ The instantaneous particle velocity vectors on contours of dimensionless enstrophy; $(b),(d)$ and $(f)$ the corresponding dimensionless fluid velocity vectors: $(a)$ and $(b)$ are the results using all the simulated modes, $(c)$ and $(d)$ are the results for $k_{C H} \eta=0.6$, and $(e)$ and $(f)$ are the results for $k_{C H} \eta=0.2$. The maximum values of the dimensionless enstrophy for $(a),(c)$ and (e) are 37.12, 18.39 and 2.90, respectively. Here $R_{\lambda}=65.3$.

Figure 14. ( $a$ ) The dimensionless enstrophy (maximum value $=35.57$ ), and $(b)$ the fluid velocity vectors of the instantaneous field for $K_{C L} L_{f}=2.5$. (c) The instantaneous particle velocity vectors on the contours of the filtered dimensionless energy. Here $R_{\lambda}=65.3$. 

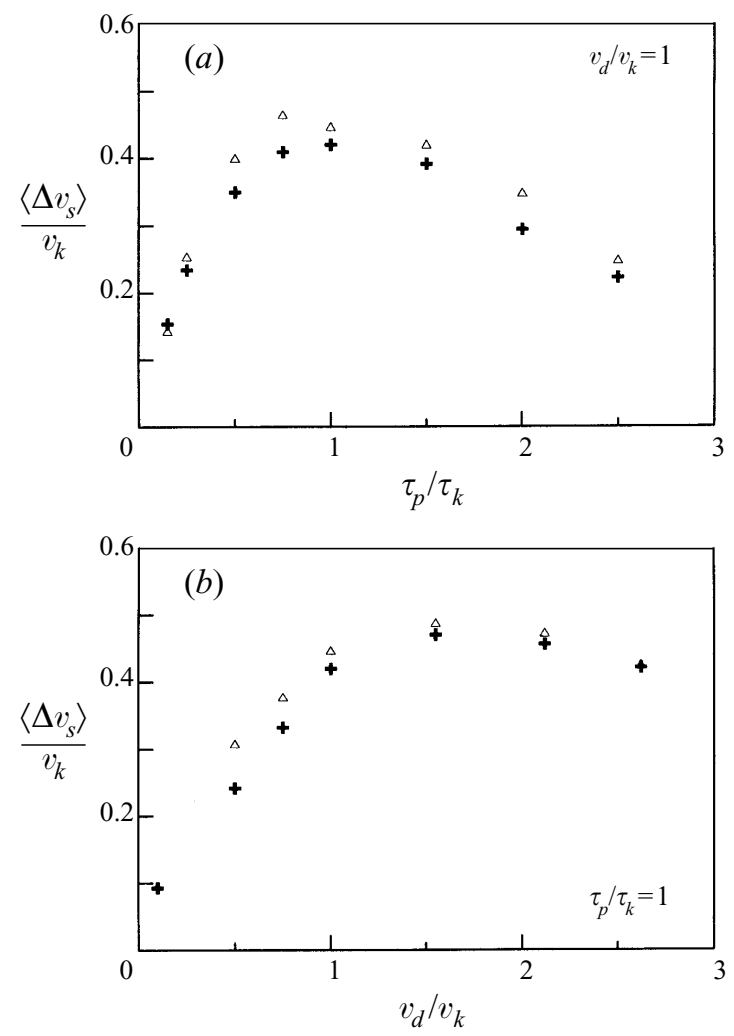

FIGURE 11. Comparison of $\left\langle\Delta v_{S}\right\rangle$ between the result using DNS $\left(96^{3}\right.$ grids, $R_{\lambda}=65.3$, denoted by $\triangle$ ) and that using LES ( $32^{3}$ grids, $R_{\lambda}=69.8$, denoted by + ).

that the cutoff wavenumber in LES, $k_{C}$, satisfies the condition $k_{C} l_{\omega}>2.5$. A result using such LES (case D), which will be called the 'extended LES' later, is shown in figure 11 together with the corresponding result using DNS (case C). Both results give essentially the same prediction of $\left\langle\Delta v_{S}\right\rangle$. The agreement for the accumulation of particles for both calculations (extended LES and DNS) is also demonstrated in figure 12 via the ensemble average of the enstrophy at the locations of the particles. Note that although the cutoff wavenumber, $k_{C}$, in the present extended LES is greater than that employed in the conventional LES, the number of grids employed here $\left(32^{3}\right)$ is still substantially less than those used in DNS $\left(96^{3}\right)$. The maximum wavenumber resolved by the extended LES is $k_{\max } \eta \approx 0.61$ (see table 1 ), while $k_{\max } \eta=1.71$ for DNS. The CPU time for the extended LES is about $10 \%$ of that for the DNS. Detailed examination of the data shows that about $95 \%$ of the total energy and $75 \%$ of the total enstrophy are resolved by the extended LES for the above case. The applicability of the extended LES also allows us to study $\left\langle\Delta v_{S}\right\rangle$ in a flow field with larger $R_{\lambda}$ which processes a short inertial subrange with $128^{3}$ grids, and is important for studying the velocity scale for $\left\langle\Delta v_{S}\right\rangle$ in $\S 3.6$.

\subsection{Effect of the large scales}

The effect of the large scales on $\left\langle\Delta v_{S}\right\rangle$ can also be studied using the above idea by chopping off the low wavenumber components. Consider the results of the particle calculations in the flow fields with $k_{C H}=k_{\max }$ and $k_{C L}>k_{\min }$. Figure 13(a) shows that $\left\langle\Delta v_{S}\right\rangle$ for the flow field with $k_{C L} L_{f}=2.5$ is about half the value of that 

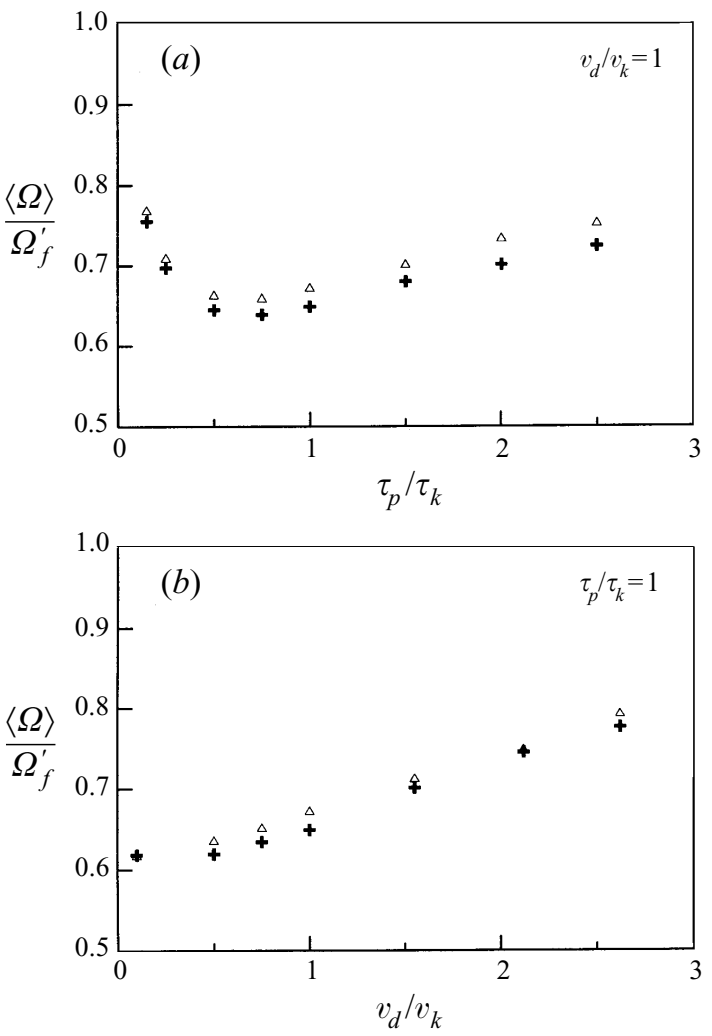

FIgURE 12. Comparison of the ensemble average of the dimensionless enstrophy at the locations of the particles between the result using DNS $\left(96^{3}\right.$ grids, $R_{\lambda}=65.3$, denoted by $\left.\triangle\right)$ and that using LES $\left(32^{3}\right.$ grids, $R_{\lambda}=69.8$, denoted by +$)$.

using all the simulated modes, and the result for $\left\langle\Delta v_{S}\right\rangle$ using the flow field with $k_{C L} L_{f}=3.25$ reduces almost to zero. Consider first the case with $k_{C L} L_{f}=2.5$. The vorticity contours in figure 14(a) (see p. 196) is almost the same as that in figure $9(a)$ (with all simulated modes). In fact, $97 \%$ of the total enstrophy has been resolved for the case using $k_{C L} L_{f}=2.5$, and the phenomenon of particle accumulation should be reasonably estimated. Although figure $13(b)$ indeed shows that the accumulation of particles in the low vorticity region for $k_{C L} L_{f}=2.5$ is similar to that for the case with all simulated modes, there still exists a certain amount of discrepancy between both results, which implies that the low wavenumber components also have moderate effects on the particle accumulation. Figure 14(b) shows the velocity field for $k_{C L} L_{f}=2.5$, which is similar to the velocity field for all simulated modes in figure $9(b)$. Detailed examination of the data shows that $84 \%$ of the energy is resolved for the case with $k_{C L} L_{f}=2.5$. In order to understand why we underestimate $\left\langle\Delta v_{S}\right\rangle$ by almost $50 \%$ with only $16 \%$ less energy in the case with $k_{C L} L_{f}=2.5$, we plot the particle velocity vector on the contours for constant values of $\Delta q / q_{\text {all }}$ in figure $14(c)$. Here $\Delta q=q_{\text {all }}-q_{2.5}$, which represents the energy contributed by the large scales of turbulence with wavenumber less than $2.5 / L_{f}$. Here $q_{\text {all }}$ and $q_{2.5}$ are the energy $\left(u_{i} u_{i} / 2\right)$ based on the calculation with all simulated modes and that with $k_{C L} L_{f}=2.5$, respectively. The result in figure 14(c) indicates that in general, more particles locate instantaneously in the high energy level as expected. According to the results in 

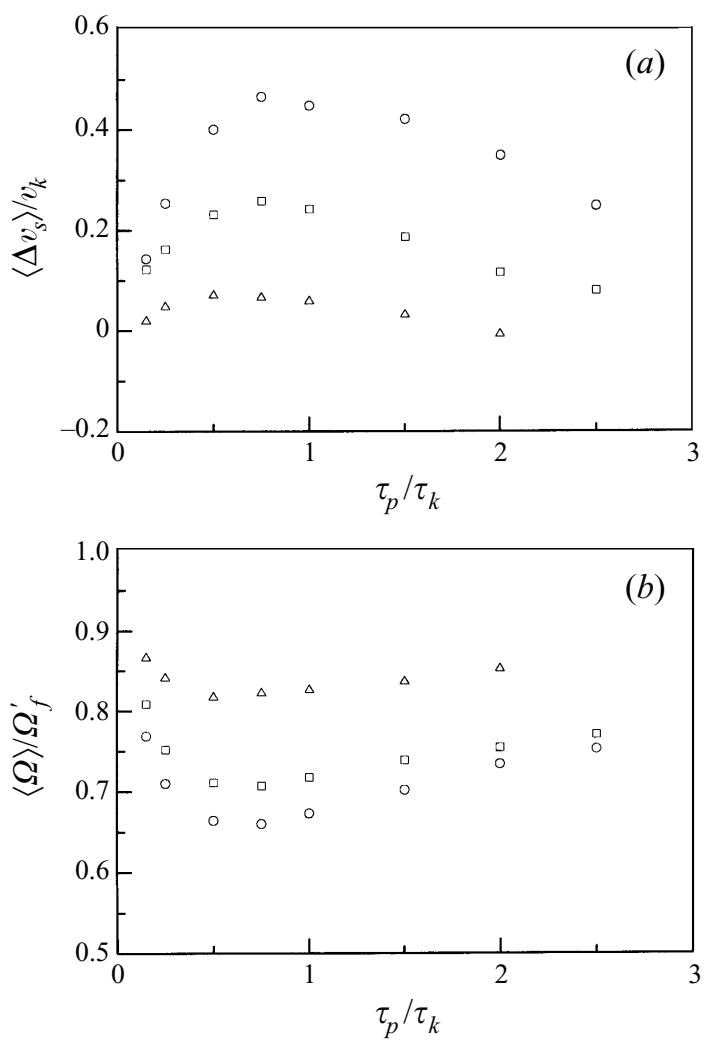

FiguRE 13. Effect of large turbulent scales on $(a)\left\langle\Delta v_{S}\right\rangle / v_{k}$, and $(b)\langle\Omega\rangle / \Omega_{f}^{\prime}$ when $R_{\lambda}=65.3$ and $v_{d} / v_{k}=1$. $O$, all simulated modes; $\square, k_{C L} L_{f}=2.5$; and $\triangle, k_{C L} L_{f}=3.25$.

figures 13 and 14, the energy of the large eddies should play a significant role on the magnitudes of the increase of the average settling velocity.

\subsection{The scales for the increase of the average settling velocity}

Although Wang \& Maxey (1993) have demonstrated the importance of Kolmogorov scaling for the processes of particle accumulation and the average settling velocity, the velocity scale for $\left\langle\Delta v_{S}\right\rangle$ deserves further study. The results in $\$ \$ 3.4$ and 3.5 show that although the particle accumulation is controlled mainly by the small scales with maximum vorticity, the average settling rate, $\left\langle\Delta v_{S}\right\rangle$, depends strongly on the large energetic scales. The variations of $\left\langle\Delta v_{S}\right\rangle / v_{k}$ with $\tau_{p} / \tau_{k}$ (or $v_{d} / v_{k}$ ) and $R_{\lambda}$ at fixed value of $v_{d} / v_{k}$ (or $\tau_{p} / \tau_{k}$ ) in figure 4 show that the data spread widely apart for different $R_{\lambda}$. Also shown in figure $4(b)$ is that the peak value of the data for a given $R_{\lambda}$ shifts slightly rightward as $R_{\lambda}$ increases. In order to study more the details of the effect of $R_{\lambda}$ on $\left\langle\Delta v_{S}\right\rangle$, two flow fields with higher $R_{\lambda}$ (cases $\mathrm{E}$ and $\mathrm{F}$ in table 1 ) were generated by the extended LES, and employed for studying the particle motion. The results in figures $15(a)$ and $15(c)$ show that the normalized data with $v_{k}$ (the Kolmogorov velocity scale) as the velocity scale spread even further apart for a larger range of $R_{\lambda}$. However, if the velocity scale for the large energetic eddies, $u^{\prime}$, is employed instead of $v_{k}$, the results in figures $15(b)$ and $15(d)$ indicate that the data for different $R_{\lambda}$ tend to collapse together. Furthermore, although the location of the peak value of $\left\langle\Delta v_{S}\right\rangle$ at a given $R_{\lambda}$ in figure $15(a)$ shifts continuously from $v_{d} / v_{k} \approx 1.2$ when $R_{\lambda}=22.6$ 

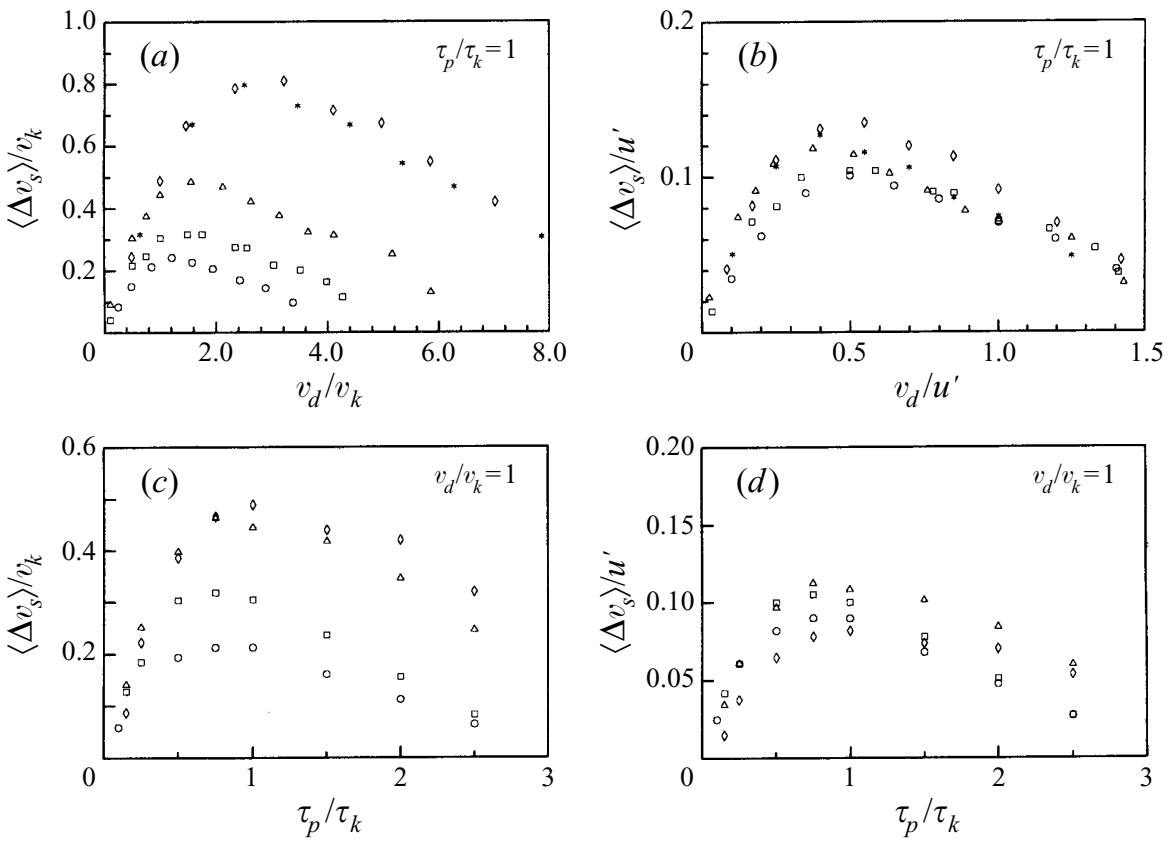

FIGURE 15. Variations of $\left\langle\Delta v_{S}\right\rangle$ with different parameters: $O, R_{\lambda}=22.6 ; \square, R_{\lambda}=35.7 ; \triangle, R_{\lambda}=65.3$; $\diamond, R_{\lambda}=133$; and $*, R_{\lambda}=153$. Here $v_{k}$ is employed as the velocity scale for $(a)$ and $(c)$, but $u^{\prime}$ is the velocity scale for $(b)$ and $(d)$.

to $v_{d} / v_{k} \approx 3.2$ when $R_{\lambda}=133$, the locations of the peak values for different $R_{\lambda}$ in figure $15(b)$ stay essentially at $v_{d} / u^{\prime} \approx 0.5$. Note that the vertical scale in figure $15(b)$ is five times greater than that in figure 15(a). Also the statistical uncertainties are quite substantial for the increase of the average settling velocity of particles (see figure 1 of Maxey 1987 for example). The small amount of scattering of the results in figure $15(b)$ is consistent with the statistical uncertainties (errors) of the calculations. A more elaborate study is carried out through a series of comprehensive calculations for the whole ranges of parameters. Figures 16(a), 16(c) and 16(e) show contours for constant values of $\left\langle\Delta v_{S}\right\rangle / v_{k}$ in $\left(v_{d} / v_{k}, \tau_{p} / \tau_{k}\right)$-planes for three different $R_{\lambda}$, which employ $v_{k}$ as the velocity scale. The corresponding results using $u^{\prime}$ as the velocity scale are shown in figures $16(b), 16(d)$ and $16(f)$. The results using $v_{k}$ as the velocity scale show that both the magnitude and the vertical location of the maximum value of $\left\langle\Delta v_{S}\right\rangle / v_{k}$ increase as $R_{\lambda}$ increases. However, the results using $u^{\prime}$ as the velocity scale show that the maximum values of $\left\langle\Delta v_{S}\right\rangle / u^{\prime}$ have essentially the same magnitude $\left(\left\langle\Delta v_{S}\right\rangle / u^{\prime}=O\left(10^{-1}\right)\right)$ and occur at almost the same location $\left(\tau_{p} / \tau_{k} \approx 1\right.$ and $v_{d} / u^{\prime} \approx 0.5$ ). Thus the more appropriate velocity scale for $\left\langle\Delta v_{S}\right\rangle$ seems to be $u^{\prime}$, the velocity scale of the large energetic eddies. There are larger discrepancies among the data in figure $15(d)$ for $v_{d} / v_{k}=1$ in comparison with those in figure $15(b)$ for $\tau_{p} / \tau_{k}=1$. This is because the results for different $R_{\lambda}$ in figure $15(d)$ correspond to the data on different horizontal straight lines with different values of $v_{d} / u^{\prime}$ in figures $16(b)$, $16(d)$ and $16(f)$. Here $v_{d} / u^{\prime}=0.415,0.331,0.244$ and 0.171 for $R_{\lambda}=22.6,35.7,65.3$, and 133 , respectively, in figure $15(d)$. If the results for a fixed value of $v_{d} / u^{\prime}$ (say, $v_{d} / u^{\prime}=0.5$ or 1 , not included in this paper) are plotted instead of those for a fixed value of $v_{d} / v_{k}$ as in figure $15(d)$, the data collapse nicely.

The reason that $u^{\prime}$ is an appropriate velocity scale and $\tau_{k}$ is an appropriate 

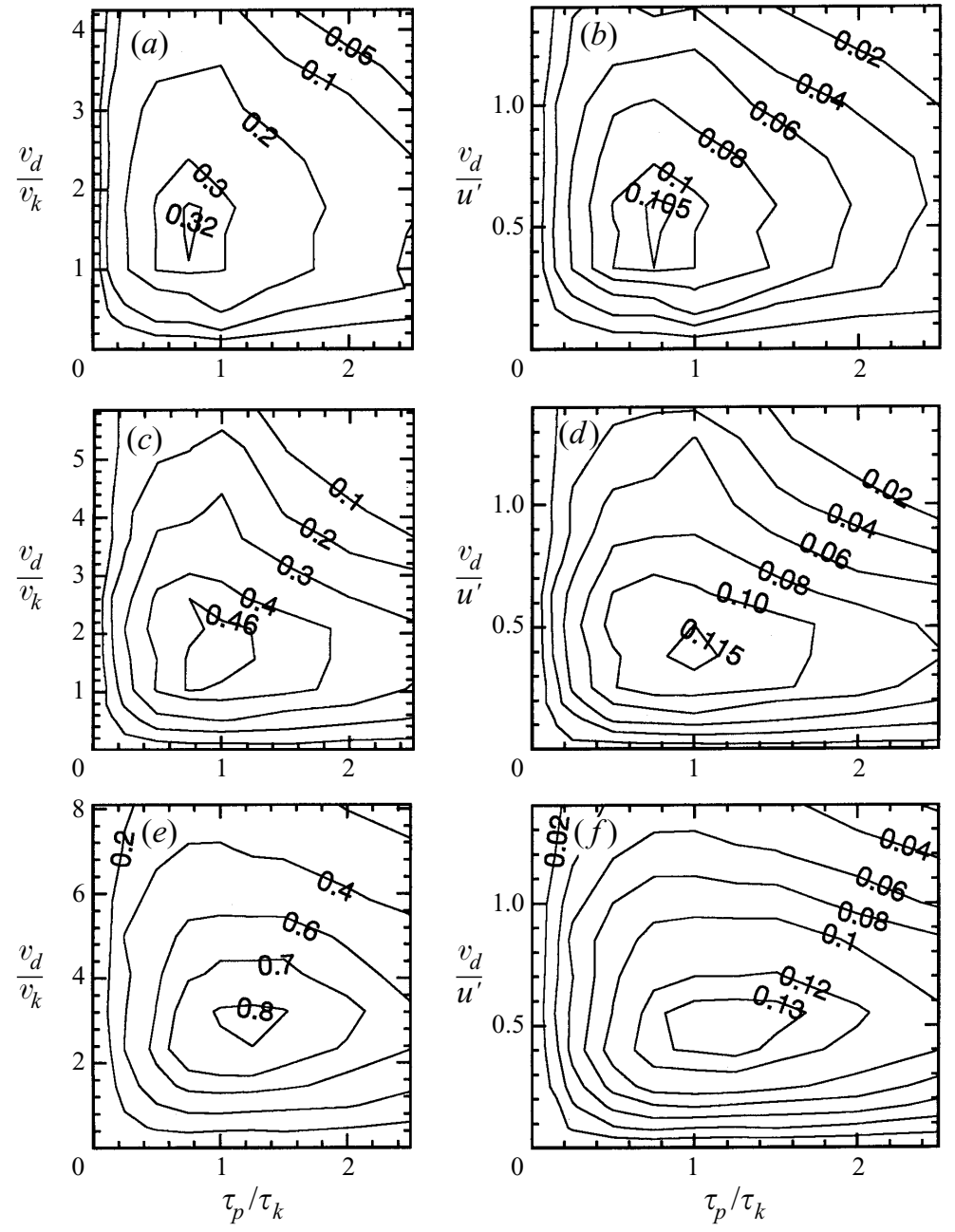

FiguRE 16. $(a),(c)$ and $(e)$ Contours for constant values of $\left\langle\Delta v_{S}\right\rangle / v_{k} .(b),(d)$ and $(f)$ Contours for constant values of $\left\langle\Delta v_{S}\right\rangle / u^{\prime}$. (a) and (b) are the results for $R_{\lambda}=35.7,(c)$ and $(d)$ are the results for $R_{\lambda}=65.3$, and $(e)$ and $(f)$ are the results for $R_{\lambda}=133$.

time scale for representing $\left\langle\Delta v_{S}\right\rangle$ may be understood as follows. According to the description of the third paragraph of $\S 1$, there are two essential elements in the increase of the average settling velocity: the particle accumulation and the drag on the particles. The accumulation of particles in the downward sweeping, low vorticity region where particles experience less local drag is a necessary condition for the increase of the average settling velocity. The increase of the average settling velocity is indeed proportional to the degree of particle accumulation since more samples are settling faster during the averaging process as discussed in $\S 3.3$. However, the magnitude of the increase of the settling velocity of an individual particle depends on the drag acting on the particle. Thus the drag on the particles is also a crucial factor in the increase of the average settling velocity of particles.

The particle accumulation is due to the centrifugal effect associated with the intense vortical structures, and is thus related to the vorticity field of the flow. The scales near the peak of the dissipation (vorticity) spectrum contribute most to the vorticity. The 
spectrum value at the peak in figure $1(b)$ is two orders greater than that at $k \eta=1$, and thus the Kolmogorov eddies can hardly play a significant role in the phenomenon of particle accumulation.

The drag on a particle is proportional to the relative velocity of the particle and the local flow field. Consider the case for small particles such that the Stokes drag law applies. If $\Delta \boldsymbol{v}=\boldsymbol{v}-\boldsymbol{v}_{d},(2 a)$ may be written as

$$
\frac{\mathrm{d} \Delta \boldsymbol{v}}{\mathrm{d} t}=\frac{\boldsymbol{u}-\Delta \boldsymbol{v}}{\tau_{p}} .
$$

As the particles tend to accumulate in the low vorticity regions where the fluid velocity has scale $u^{\prime}$ (see figure 9), it follows from (12) that $u^{\prime}$ is an appropriate scale for representing $\langle\Delta v\rangle$. More rigorously, the local fluid velocity in the wavenumber domain is expressed in terms of a finite Fourier series in the DNS (or LES) using a pseudospectral method, with each term representing the contribution from a given turbulent scale. The relative importance of each term depends on its coefficient, which is related directly to the energy spectrum. The energy of the large energetic scales is three to four orders greater than that of the Kolmogorov scales (see figure 1a), and thus the large energetic scales contribute mainly to the local fluid velocity $\boldsymbol{u}$, or the drag on the particle. The Kolmogorov scales play essentially no role on the drag and thus the increase of the average settling velocity of particles.

Thus the increase of the average settling velocity of particles in homogeneous isotropic turbulence is a physical problem involving quite a wide range of scales ranging from the large energetic scales to the small scales approximately with wavenumber $2.5 k_{\omega}$. The small scales are primarily responsible for 'moving' the particles to the regions where the local drag on a particle is less than the average value. The reduction of the drag on a given particle depends on the local fluid velocity, which is controlled mainly by the large energetic scales. Both the small scales with maximum vorticity and the large scales with maximum energy are crucial for the increase of the average settling velocity of particles. The Kolmogorov scales play essentially no dynamical role on the problem because both their vorticity and energy are negligible. The separation between the wavenumber corresponding to the peak of the dissipation (vorticity) spectrum and that of the energy spectrum increases as $R_{\lambda}$ increases. When $R_{\lambda}$ is sufficiently large, the large scales have an insignificant effect on the particle accumulation, but the drag on every individual particle still depends strongly on the large energetic scales. Thus there are certain physical reasons for supporting the choice of $u^{\prime}$ as the velocity scale for representing the increase of the average settling velocity.

It is interesting that the Kolmogorov time scale, $\tau_{k}$ appears as an approximate time scale for the present problem. Note that the results in figures 15 and 16 are maximized at $\tau_{p} / \tau_{k} \approx 1$. As the small scales of turbulences with wavenumber greater than $2.5 k_{\omega}$ are found to have a negligible effect on $\left\langle\Delta v_{S}\right\rangle$ in $\S 3.4$, the entry of $\tau_{k}$ is thus considered not to be related directly to the Kolmogorov eddies. A plausible way that $\tau_{k}$ might play a significant role in the problem is through the phenomenon of particle accumulation. Since the scale for the vorticity associated with the 'eddies' with wavenumber $k_{\omega}$ is of order $u^{\prime} / \lambda$ (see Tennekes \& Lumley 1972), which equals $(15)^{-0.5} \tau_{k}^{-1}$, and the scales near $k_{\omega}$ are responsible mainly for the particle accumulation, $\tau_{k}$ thus appears as the appropriate time scale of the present problem.

The phenomenon of the increase of the average settling velocity due to the turbulence occurs for limited ranges of $v_{d} / u^{\prime}$ and $\tau_{p} / \tau_{k}$ according to the above result. If this result can be extended to cases with larger values of $R_{\lambda}$, we may examine its effect for some applications. As $v_{d}=g \tau_{p} / C_{0}$ (see $\S 2.1$ ), the particle free fall velocity $v_{d}$ is 
related to the particle's relaxation time $\tau_{p}$ for a given body force $\boldsymbol{g}$. Consider first the gravitational settling of sand particles in the atmospheric boundary layer. On taking $\rho_{p} / \rho \approx 1700, v \approx 1.5 \times 10^{-5} \mathrm{~m}^{2} \mathrm{~s}^{-1}, u^{\prime} \approx 0.5 \mathrm{~m} \mathrm{~s}^{-1}$ and $l \approx 200 \mathrm{~m}$ (see Lugt 1983), where $l$ is the size of the large eddies, we found $\epsilon \approx u^{\prime 3} / l \approx 6.25 \times 10^{-4} \mathrm{~m}^{2} \mathrm{~s}^{-3}, \eta \approx 1.52$ $\mathrm{mm}, \tau_{k} \approx 0.155 \mathrm{~s}$ and $R_{\lambda} \approx 10^{4}$. If $v_{d}$ is chosen to be $0.5 u^{\prime}$, we found $d_{p} \approx 68 \mu \mathrm{m}$ and $\tau_{p} \approx 0.0292 \mathrm{~s}$. It follows that $\tau_{p} / \tau_{k} \approx 0.188$, which is much less than the unity required for the increase of the average settling velocity to be significant (see figure 16f). However, the phenomenon could be pronounced for some engineering applications. Consider for example the gravitational settling of oil drops in the combustion chamber of a power plant. Due to the high temperature environment, we set $\rho_{p} / \rho \approx 2000$ and $v \approx 10^{-4} \mathrm{~m}^{2} \mathrm{~s}^{-1}$. On taking $u^{\prime} \approx 1 \mathrm{~m} \mathrm{~s}^{-1}$ and $l \approx 10 \mathrm{~m}$, we found $\epsilon \approx 0.1 \mathrm{~m}^{2} \mathrm{~s}^{-3}$, $\eta \approx 1.78 \mathrm{~mm}, \tau_{k} \approx 0.0316 \mathrm{~s}$ and $R_{\lambda} \approx 1.2 \times 10^{3}$. If $v_{d}$ is chosen to be $0.5 u^{\prime}$, we found $d_{p} \approx 229 \mu \mathrm{m}$ and $\tau_{p} \approx 0.0583 \mathrm{~s}$. It follows that $\tau_{p} / \tau_{k} \approx 1.85$, which is near unity for $\left\langle\Delta v_{S}\right\rangle$ to be maximized. For some smaller combustion chambers, $u^{\prime} \approx 0.5 \mathrm{~m} \mathrm{~s}^{-1}$ and $l \approx 1 \mathrm{~m}$, we found $R_{\lambda} \approx 270$ and $\tau_{p} / \tau_{k} \approx 0.96$ if $v_{d} / u^{\prime} \approx 0.5$, which falls into the parameter ranges where the increase of the average settling velocity is significant.

\section{Conclusions}

When heavy solid particles settle under a body force field in homogeneous isotropic turbulence, the particles tend to accumulate in the downward sweeping, low vorticity regions (where the local drag on a particle is less than the average value) due to the local centrifugal effect associated with the intense tubular vortical structures. The accumulation effect is necessary for the increase of the average settling velocity, and is controlled mainly by the small scales of order $l_{\omega}$, which equals the inverse of the wavenumber corresponding to the maximum of the dissipation (vorticity) spectrum. However, the increase of the average settling rate depends also on the drag on an individual particle, which is controlled mainly by the large energetic scales. The Kolmogorov scales, which are in general of one order less than $l_{\omega}$, have essentially no effect on either the particle accumulation (because of their relative weak vorticity) or the drag on the particles (because of their lack of energy), and thus contribute no role in the increase of the average settling rate of particles. The flow field generated by the large eddy simulation is adequate for studying the present problem, provided the cutoff wavenumber in the simulation is greater than $2.5 / l_{\omega}$. This is an interesting example for turbulent transport where both the large and small scales (but not the smallest (Kolmogorov) scales) of turbulence are important for the phenomenon.

Through a series of calculations for different parameters, the increase of the average settling rate in stationary turbulence is found to be maximized and of order $u^{\prime} / 10$ when $v_{d} \approx 0.5 u^{\prime}$ and $\tau_{p} / \tau_{k} \approx 1$ for $R_{\lambda}=22.6-153$, which implies that the appropriate velocity and time scales for the present problem are $u^{\prime}$ and $\tau_{k}$, respectively. Here $u^{\prime}$ is the velocity scale associated with the large energetic eddies, which are primarily responsible for the magnitude of $\left\langle\Delta v_{S}\right\rangle$, and $\tau_{k}^{-1}$ is the scale for the vorticity of the 'eddies' with size $l_{\omega}$, which are associated with the accumulation of the particles in the low vorticity region. The settling of particles in homogeneous isotropic decaying turbulence was also studied by the present authors (see Yang 1997), and the behaviour of the average settling velocity in decaying turbulence is qualitatively similar to that in stationary turbulence.

The present study is supported partially by the National Science Council of the Republic of China through the grant NSC86-2212-E-002-074. The authors are also 
grateful to the National Center for High-performance Computing for the free computer time of using the Cray C90 computer.

\section{REFERENCES}

Clift, R., Grace, J. R. \& Weber, M. E. 1978 Bubbles, Drops and Particles. Academic Press.

Eswaran, E. \& Pope, S. B. 1988 An examination of forcing in direct numerical simulations of turbulence. Comput. Fluids 16, 257-278.

Ferziger, J. H. 1983 Higher-level simulations of turbulent flows. In Computational Methods for Turbulent, Transonic and Viscous Flows (ed. J. A. Essers) pp. 93-182. Hemisphere.

Jiménez, J., Wary, A., Saffman, P. G. \& Rogallo, R. S. 1993 The structure of intense vorticity in isotropic turbulence. J. Fluid Mech. 255, 65-90.

LeE, M. J. \& REYNolds, W. C. 1985 Numerical experiments on the structure of homogeneous turbulence. Rep. TF-24. Mech. Eng. Dept., Stanford University.

Lugt, H. J. 1983 Vortex Flow in Nature and Technology. John Wiley \& Sons.

MAXEY, M. R. 1987 The gravitational settling of aerosol particles in homogeneous turbulence and random flow field. J. Fluid Mech. 175, 441-465.

McMillian, O. J. \& Ferziger, J. H. 1979 Direct testing of subgrid scale models. AIAA J. 17, 1340-1346.

Patterson, G. S. \& Orszag, S. A. 1971 Spectral calculations of isotropic turbulence: Efficient removal of aliasing interactions. Phys. Fluids 14, 1538-1541.

REEKs, M. W. 1977 On the dispersion of small particles suspended in an isotropic turbulent fluid. J. Fluid Mech. 83, 529-546.

Riley, J. J. \& Patterson, G. S. 1974 Diffusion experiments with numerically integrated isotropic turbulence. Phys. Fluids 17, 292-297.

RogaLLO, R. S. 1977 An ILLIAC program for the numerical simulation of homogeneous turbulence. NASA Tech. Mem. 73203.

Rogallo, R. S. 1981 Numerical experiments in homogeneous turbulence. NASA Tech. Mem. 81315.

SQuires, K. D. \& EATON, J. K. 1991 Preferential concentration of particles by turbulence. Phys. Fluids A 3, 1169-1178.

Tennekes, C. M. \& Lumley, J. L. 1972 A First Course in Turbulence. MIT Press.

Townsend, A. A. 1951 On the fine-scale structure of turbulence. Proc. R. Soc. Lond. A 208, 534-542.

Vincent, A. \& MENEGUZZI, M. 1991 The spatial structure and statistical properties of homogeneous turbulence. J. Fluid Mech. 225, 1-20.

WANG, L.-P. \& MAXEY, M. R. 1993 Settling velocity and concentration distribution of heavy particles in homogeneous isotropic turbulence. J. Fluid Mech. 256, 27-68.

YANG, C. Y. 1997 Settling of heavy particles in homogeneous turbulence. PhD dissertation, National Taiwan University.

YEH, F. \& LEI, U. 1991 On the motion of small particles in a homogeneous isotropic turbulent flow. Phys. Fluids A 3, 2571-2586.

Yeung, P. K. \& Pope, S. B. 1988 An algorithm for tracking fluid particles in numerical simulations of homogeneous turbulence. J. Comput. Phys. 79, 373-415. 